\title{
The theory of minimal surfaces in $M \times \mathbb{R}$
}

\author{
William H. Meeks and Harold Rosenberg*
}

\begin{abstract}
In this paper, we develop the theory of properly embedded minimal surfaces in $M \times \mathbb{R}$, where $M$ is a closed orientable Riemannian surface. We construct many examples of different topology and geometry. We establish several global results. The first of these theorems states that examples of bounded curvature have linear area growth, and so, are quasiperiodic. We then apply this theorem to study and classify the stable examples. We prove the topological result that every example has a finite number of ends. We apply the recent theory of Colding and Minicozzi to prove that examples of finite topology have bounded curvature. Also we prove the topological unicity of the embedding of some of these surfaces.
\end{abstract}

Mathematics Subject Classification (2000). Primary 53A10; Secondary 49Q05, 53C42.

Keywords. Minimal surface, flux, stable minimal surface, index of stability, minimal lamination, curvature estimates, periodic minimal surface, quasiperiodic.

\section{Introduction}

In the present paper, we begin the development of a general theory for properly embedded minimal surfaces $\Sigma$ in a Riemannian three-manifold of the form $M \times \mathbb{R}$, where $M$ is a closed orientable Riemannian surface. The theorems stated in this Introduction form the main body of this emerging theory. We state once more for emphasis that in all of these theorems and throughout this paper, $M$ will denote a closed orientable Riemannian surface. We will consider only non-compact minimal surfaces $\Sigma$. For, if $\Sigma$ is compact, then $\Sigma=M \times\{t\}$ for some $t \in \mathbb{R}$, since the height function on $M \times \mathbb{R}$, restricts to a harmonic function on $\Sigma$ which has a maximum on $\Sigma$, and hence is constant.

In Section 2, we shall construct a multitude of examples of $\Sigma$ in $M \times \mathbb{R}$ of varying topology and geometry, comparable to the rich family of classical doubly periodic minimal surfaces in $\mathbb{R}^{2} \times \mathbb{R}$ considered to be properly embedded minimal surfaces $\Sigma$

\footnotetext{
*This material is based upon work by the NSF under Award No. DMS - 0405836. Any opinions, findings, and conclusions or recommendations expressed in this publication are those of the authors and do not necessarily reflect the views of the NSF.
} 
in the quotient $\mathbb{T} \times \mathbb{R}$, where $\mathbb{T}$ is a flat two dimensional torus. In $S \times \mathbb{R}$, where $S$ is the two-sphere of constant curvature one, we have helicoids obtained by spinning a geodesic of $S \times\{0\}$ while rising in the $\mathbb{R}$ factor at a speed proportional to the constant rate of spinning. The surfaces of revolution in $S \times \mathbb{R}$ are the unduloids, which behave qualitatively like the Delaunay unduloids of constant mean curvature $\frac{1}{2}$ in $\mathbb{R}^{3}$. We construct a two-parameter family $\mathcal{A}$ of properly embedded minimal annuli in $S \times \mathbb{R}$, containing the helicoids and the unduloids, and each of these annuli meets the level set spheres $S \times\{t\}$ in circles with varying radii. The annuli in $\mathcal{A}$ are parametrized by the same family of elliptic functions as the Abresch two-parameter family [1] of constant mean curvature $\frac{1}{2}$ periodic annuli in $\mathbb{R}^{3}$ foliated by planar lines of curvature (the larger principal curvatures). We also construct many other interesting $\Sigma$ of finite topology in $M \times \mathbb{R}$, including an infinite number of complete minimal graphs over non-isotopic domains in $M$, when the genus of $M$ is greater than one (see Theorem 2.3 in Section 2).

Our first main theoretical result deals with surfaces with bounded curvature.

Theorem 1.1 (Linear Area Growth Theorem). If $\Sigma$ is a properly embedded connected non-compact minimal surface in $M \times \mathbb{R}$ of bounded curvature, then $\Sigma$ has linear area growth, in the sense that given any $t \geq 1$, then $c_{1} t \leq \operatorname{Area}(\Sigma \cap(M \times[-t, t])) \leq c_{2} t$, for some positive constants $c_{1}, c_{2}$. Here $c_{1}$ depends only on $M$ and $c_{2}$ depends on $M$, an upper and lower bound of the flux of the harmonic height function $h: \Sigma \rightarrow \mathbb{R}$ across a level set of $h$, an upper bound on the absolute Gaussian curvature of $\Sigma$ and on the homology class represented by the cycle $\Sigma \cap(M \times\{0\})$ in $H_{1}(M \times\{0\})$.

An immediate corollary of Theorem 1.1 is the following:

Corollary 1.1. If $\Sigma$ is a properly embedded minimal surface of bounded curvature in $M \times \mathbb{R}$, then $\Sigma$ has a finite number of ends.

Note that if $\Sigma$ separates $M \times \mathbb{R}$, then $\Sigma \cap(M \times\{0\})$ represents the zero homology class in $H_{1}(M \times\{0\})$, and so, in this case the constant $c_{2}$ in the above theorem depends only on $M$, curvature estimates for $\Sigma$ and a lower bound for the flux of $\Sigma$. In particular, if $M$ is a two-sphere, then we get this simpler estimation for $c_{2}$.

Every sequence of properly embedded minimal surfaces in a Riemannian threemanifold, which satisfy uniform local area and local curvature estimates and such that each surface in the sequence intersects some compact domain, has a subsequence that converges to a properly embedded minimal surface with the same uniform local area and local curvature estimates (see for example [27] and [38]). A simple consequence of this compactness result and Theorem 1.1 is that every non-compact properly embedded minimal surface in $M \times \mathbb{R}$ with bounded curvature is quasiperiodic in the following sense. A properly embedded surface $\Sigma$ in a Riemannian three-manifold $N$ is quasiperiodic, if there exists a discrete infinite closed subset $S=\left\{T_{n} \mid n \in \mathbb{N}\right\}$ 
of the isometry group of $N$ such that $T_{n}(\Sigma)$ converges on compact subsets of $N$ to a properly embedded surface.

Corollary 1.2. If $\Sigma$ is a properly embeddednon-compact minimal surface of bounded curvature in $M \times \mathbb{R}$, then $\Sigma$ is quasiperiodic. In fact, any sequence of vertical translations of $\Sigma$ in $M \times \mathbb{R}$ contains a convergent subsequence to another properly embedded minimal surface with the same bound on its curvature.

In Section 4, we study stable minimal surfaces in $M \times \mathbb{R}$. By the curvature estimates of Schoen [43], every properly embedded stable minimal surface in $M \times \mathbb{R}$ has bounded curvature. (Schoen's theorem states that any immersed complete stable orientable minimal surface in a homogenous regular three-manifold has uniform curvature estimates away from its boundary. Thus, if it has empty boundary, then it has bounded curvature. In our case, if $\Sigma$ were non-orientable, stable and properly embedded in $M \times \mathbb{R}$, then small balls in $M \times \mathbb{R}$ intersected with $\Sigma$ are compact surfaces that separate the ball, and so, Schoen's curvature estimates imply $\Sigma$ has bounded curvature.) Therefore, every properly embedded non-compact stable minimal surface in $M \times \mathbb{R}$ is quasiperiodic. This quasiperiodicity property will be essential in proving the following theorem, which we refer to as the Stability Theorem and whose proof appears in [26]. This result is Theorem 1.1 in [26]. This major result classifies all the non-compact properly embedded orientable stable minimal surfaces into three categories: they are surfaces of the form $\gamma \times \mathbb{R}$ where $\gamma$ is a simple closed stable geodesic in $M$, minimal graphs over domains in $M \times\{0\}$, or the periodic multigraphs $M(\alpha, r)$ described in Theorem 4.1. The proof of the Stability Theorem is based in part on the theoretical results we obtain in Sections 3 and 4 of this paper.

Theorem 1.2 (Stability Theorem (Theorem 1.1 in [26])). Suppose that $\Sigma$ is a connected properly embedded stable orientable minimal surface in $M \times \mathbb{R}$. Then, $\Sigma$ is one of the surfaces described in (1)-(4) below.

(1) $\Sigma$ is compact and $\Sigma=M \times\{t\}$ for some $t \in \mathbb{R}$.

(2) $\Sigma$ is of the form $\gamma \times \mathbb{R}$, where $\gamma$ is a simple closed stable geodesic in $M$.

(3) $\Sigma$ is periodic under some vertical translation by height $r$, and so has a quotient $\bar{\Sigma}$ in $M \times S(r)$, where $S(r)$ is a circle of circumference $r$. In this case, for every $p \in M,\{p\} \times S(r)$ intersects $\bar{\Sigma}$ transversely in a single point and the orbit of the natural action of $S(r)$ on $M \times S(r)$ gives rise to a product minimal foliation of $M \times S(r)$. In particular, $\bar{\Sigma}$ is homeomorphic to $M$ and is area minimizing in its integer homology class.

(4) $\Sigma$ is a graph over an open connected subdomain of $M$ bounded by a finite number of stable geodesics, with each end of $\Sigma$ asymptotic to the end of one of the flat vertical annuli described in (2). 
(5) The moduli space of examples described in (3) in the case $M$ is orientable is naturally parametrized by $P\left(H_{1}(M)\right) \times \mathbb{R}^{+}$, where $P\left(H_{1}(M)\right)$ consists of the primitive (non-multiple) elements in the first homology group of $M$.

Corollary 1.1 states that a properly embedded minimal surface of bounded curvature in $M \times \mathbb{R}$ must have a finite number of ends. In Section 5, we prove that the bounded curvature hypothesis on the surface can be dropped and one still obtains the finite number of ends conclusion.

Theorem 1.3 (Finiteness of Ends Theorem). If $\Sigma$ is a properly embedded minimal surface in $M \times \mathbb{R}$, then $\Sigma$ has a finite number of ends.

Corollary 1.3. If $\Sigma$ is a properly embedded minimal surface of finite genus in $M \times \mathbb{R}$, then $\Sigma$ is conformally a closed Riemann surface punctured in a finite number of points.

The above Finiteness of Ends Theorem appears to be the only interesting general result which restricts the topological structure of a properly embedded minimal surface $\Sigma$ in $M \times \mathbb{R}$. However, for particular metrics on $M$, one can obtain deeper results on the topology and on the embedding of $\Sigma$ in $M \times \mathbb{R}$. The next theorem, whose proof appears in Section 6, gives an important example of such a result.

Recall that a handlebody is a three-manifold with boundary that is homeomorphic to a closed regular neighborhood of a connected properly embedded one dimensional $\mathrm{CW}$ complex in $\mathbb{R}^{3}$ and that a surface $\Sigma$ in a three-manifold $N^{3}$ is a Heegaard surface if it separates $N^{3}$ into closed complements which are handlebodies. Rosenberg [41] proved that a properly embedded minimal surface in $S \times \mathbb{R}$ must have exactly two ends, where $S$ is the two-sphere endowed with a metric of positive Gaussian curvature. The next theorem gives a deep generalization of this result to a larger class of metrics on $S$.

Theorem 1.4 (Unknotted Theorem). Suppose $S$ is a two-sphere endowed with a Riemannian metric with no stable simple closed geodesics. Then:

(1) If $\Sigma$ is a non-compact properly embedded minimal surface in $S \times \mathbb{R}$, then $\Sigma$ is a Heegaard surface for $S \times \mathbb{R}$.

(2) Every Heegaard surface for $S \times \mathbb{R}$ has two ends, and if $\Sigma$ is a connected orientable surface with two ends, then $\Sigma$ embeds in $S \times \mathbb{R}$ as a Heegaard surface.

(3) Heegaard surfaces of $S \times \mathbb{R}$ are unknotted, in the sense that if two such surfaces $M_{1}, M_{2}$ are diffeomorphic, then there exists an orientation preserving diffeomorphism $f \in \operatorname{Diff}(S \times \mathbb{R})$ such that $f\left(M_{1}\right)=M_{2}$. 
We next focus our attention on the case when the properly embedded minimal surface $\Sigma$ in $M \times \mathbb{R}$ has finite genus. By Theorem 1.3, such a surface $\Sigma$ has a finite number of ends, and so, each end of $\Sigma$ is an annulus and $\Sigma$ has finite topology. We then use this finite topology property of $\Sigma$ to prove that $\Sigma$ has bounded curvature, and so, by Theorem 1.1, $\Sigma$ has linear area growth. The proof that $\Sigma$ has bounded curvature uses some of the recent results of Meeks, Perez and Ros [20] on the local structure of properly embedded minimal surfaces with bounded genus in three-manifolds in a neighborhood of a point of large curvature. Their results in turn depend on recent curvature estimates of Colding and Minicozzi [2], [3], [5], work of Meeks [35], and results of Meeks and Rosenberg in [27]. With some further geometric analysis, we obtain the following two theorems in Section 7.

Theorem 1.5 (Bounded Curvature Theorem). Suppose $\Sigma$ is a properly embedded minimal surface of finite genus in $M \times \mathbb{R}$. Then:

(1) $\Sigma$ has bounded curvature. Hence, by Theorem 1.1, it has linear area growth and by Corollary 1.3 it has finite conformal type.

(2) If $M$ has non-positive curvature, then $\Sigma$ has finite index with respect to the stability operator and total curvature $c(\Sigma)=2 \pi \chi(\Sigma)$.

(3) If $M$ has non-positive curvature and $M$ is not a torus, then each end of $\Sigma$ is asymptotic to $\gamma \times \mathbb{R}$, where $\gamma$ is a stable embedded geodesic in $M$. When $M$ is a flat torus, then each end of $\Sigma$ is asymptotic to the end of a totally geodesic flat annulus in $M \times \mathbb{R}$.

(4) If $\Sigma(n)$ is a sequence of properly embedded minimal annuli in $M \times \mathbb{R}$ with diverging curvature, then the fluxes of the $\Sigma(n)$ converge to zero. (Here, the flux of $\Sigma(n)$ refers to the flux of the gradient of the harmonic height function $h: \Sigma \rightarrow \mathbb{R}$ across a level set of $h$.)

(5) Suppose $M$ is not topologically the two-sphere. Then, there exists an $\varepsilon>0$ such that if $\Sigma$ is a properly embedded unstable minimal annulus in $M \times \mathbb{R}$, then the flux of $\Sigma$ is at least equal to $\varepsilon$. In particular, there exists a positive constant $C_{M}$ so that any properly embedded minimal annulus in $M \times \mathbb{R}$ has absolute curvature bounded by $C_{M}$. When $\Sigma$ is a properly embedded minimal surface of finite topology in $M \times \mathbb{R}$, then outside of a compact set it has curvature bounded by $2 C_{M}$.

Theorem 1.6. Suppose $\Sigma$ is a properly embedded minimal surface in $M \times \mathbb{R}$. If there exists an $\varepsilon>0$, such that for every $a \in \mathbb{R}$, the genus of $\Sigma \cap(M \times[a-\varepsilon, a+\varepsilon])$ is bounded independent of a, then $\Sigma$ has linear area growth.

At the end of the paper, we include a section on concluding remarks and conjectures. 
The theorems stated in this Introduction are strong results on the geometric behavior of minimal surfaces $\Sigma$ of finite topology in $M \times \mathbb{R}$, which significantly generalize our earlier theorems [23], where $M=\mathbb{T}$ is a flat two dimensional torus. One of the main theorems in [23] is that such a $\Sigma$ has bounded curvature and total Gaussian curvature $c(\Sigma)=2 \pi \chi(\Sigma)$, where $\chi(\Sigma)$ is the Euler characteristic of $\Sigma$; thus, $\Sigma$ has finite total curvature if it has finite topology. This finite total curvature property for finite topology $\Sigma$ leads to strong restrictions on the geometry and the topology of such surfaces and forces each annular end of $\Sigma$ to be asymptotic to the end of a flat annulus in $\mathbb{T} \times \mathbb{R}$.

Later Meeks (see Theorem 5.2 in [32]) generalized these results in [23] by proving that any properly embedded minimal surface $\Sigma$ in $\mathbb{T} \times \mathbb{R}$ has a finite number of ends and by proving that if the genus of $\Sigma$ is finite, then $\Sigma$ has finite topology and linear area growth. Meeks' Theorems identified properly embedded minimal surfaces in $\mathbb{T} \times \mathbb{R}$ of finite total curvature with those surfaces of finite genus. In particular, if $\Sigma$ has genus zero, then $\Sigma$ has finite total curvature. This result, together with some other constraints finite total curvature planar domains in $\mathbb{T} \times \mathbb{R}$ satisfy [23], was then applied by Lazard-Holly and Meeks [16] to prove the deep result: A genus zero quotient of a properly embedded doubly-periodic minimal surfaces in $\mathbb{R}^{3}$ is the quotient of one of the classical doubly-periodic examples defined by Scherk [42] in 1835 (also see [15]).

The authors would like to thank Joaquin Perez for his careful reading of a preliminary version of this manuscript and for his numerous and detailed suggestions for improving it. We would like to thank him as well for making the Figure 1 in Section 2.

\section{Constructing examples of finite topology}

In this section, we will cover some methods that have proven useful in constructing properly embedded minimal surfaces $\Sigma$ in $M \times \mathbb{R}$ of finite topology. First, note that the height function $h: \Sigma \rightarrow \mathbb{R}$ is harmonic, and so, by the maximum principle, if $\Sigma$ is compact, then it is a level set surface of the form $M \times\{t\}$ for some $t \in \mathbb{R}$. Since $M \times \mathbb{R}$ has two ends, a non-compact $\Sigma$ must have at least two ends, again by a simple application of the maximum principle. Thus, the simplest non-compact examples are minimal annuli $A \subset M \times \mathbb{R}$. Since $h: A \rightarrow \mathbb{R}$ is proper on each end of $A, A$ can be conformally parametrized by $S(r) \times \mathbb{R}$ where $h^{-1}(t)=S(r) \times\{t\}$ and $S(r)$ is the circle of circumference $r$, where $r$ is the flux of $A$ (the flux of $A$ is defined in the first paragraph of Section 3 and is just the flux of the gradient of $h$ across any level set). In particular, $A$ intersects each level set $M \times\{t\}$ transversely in a simple closed curve.

2.1. A minimax construction for minimal annuli in $S \times \mathbb{R}$. The simplest noncompact properly embedded minimal annuli in $M \times \mathbb{R}$ are the totally geodesic annuli 
obtained as $\gamma \times \mathbb{R}$, where $\gamma$ is a simple closed geodesic on $M$. If $\gamma$ is an unstable geodesic, then frequently there exists a one-parameter family $A(t)$ of periodic properly embedded minimal annuli that are unstable (infinite index) and are small (horizontal) graphs over $\gamma \times \mathbb{R}$. In [37], Pedrosa and Ritore described analytically what these annuli are in the case that $M$ is a two-sphere $S$ endowed with a constant curvature metric and they called them unduloids. One can also define helicoids [41] in $S \times \mathbb{R}$; they are obtained by rotating a great circle on $S$ at a constant rate in the $t$ parameter about an axis passing through a pair of antipodal points on the rotated great circle. Our first step in constructing other properly embedded minimal annuli in $S \times \mathbb{R}$ is the following lemma, whose proof we postpone to the end of the subsection.

Lemma 2.1. Let $S$ denote the unit two-sphere in $\mathbb{R}^{3}$. Let $\alpha$ denote the great circle on $S$ which is the intersection of $S$ with the $x_{1} x_{3}$-plane and let $\alpha(0)=\alpha \times\{0\}$. For $\theta$, $0<\theta<\pi$, let $\alpha(\theta)$ denote the great circle obtained by rotating $\alpha$ counterclockwise by angle $\theta$ around the positively oriented $x_{3}$-axis. For $t>0$, let $\alpha(\theta, t)$ denote the great circle $\alpha(\theta) \times\{t\} \subset S \times \mathbb{R}$. Define the helicoid $W(\theta, t)=\bigcup\left\{\alpha\left(\frac{s \theta}{t}, s\right) \mid 0 \leq\right.$ $s \leq t\}$ with boundary $\alpha(0)$ and $\alpha(\theta, t)$. For $\theta$ fixed and for $t$ sufficiently small such that $W(\theta, t)$ is strictly stable, there exists an embedded index one minimal annulus $A(\theta, t) \subset S \times[0, t]$ with $\partial(A(\theta, t))=\alpha(0) \cup \alpha(\theta, t)$, which is not a helicoid and such that the interior of $A(\theta, t)$ is disjoint from $W(\theta, t)$. Furthermore, $A(\theta, t)$ has the symmetries of the union of its boundary circles.

Let $A(\theta, t)$ be one of the compact minimal annuli in the previous lemma. This annulus is part of the periodic minimal annulus $\widetilde{A}(\theta, t)$ in $S \times \mathbb{R}$ obtained as the orbit of the subgroup of the isometry group of $S \times \mathbb{R}$ generated by reflection across each of the boundary curves of $A(\theta, t)$; note that the reflection across $\alpha \subset S \times\{0\}$ is the composition of reflection across $S \times\{0\}$ composed with the reflection in the vertical annulus $\alpha \times \mathbb{R}$. Also, $\widetilde{A}(\theta, t)$ is invariant under reflection across $\gamma \times \mathbb{R}$, where $\gamma$ is the equator in $S$, and it is invariant under reflection across a great circle in $S \times\left\{\frac{t}{2}\right\}$.

We now check that for $t$ sufficiently small, $\widetilde{A}(\theta, t)$ is not an unduloid and not a helicoid. Since $\widetilde{A}(\theta, t)$ contains the non-parallel great circles $\alpha(0)$ and $\alpha(\theta, t)$, it is not an unduloid. $A(\theta, t)$ is not a helicoid, since the level curves in the interior are not geodesics; they are disjoint from the geodesic level curve of $W(\theta, t)$ at this level. This completes the proof of the next theorem, where $t=t(\theta)$ is chosen small enough so that $W(\theta, t)$ is strictly stable.

Theorem 2.1. For every $\theta, 0<\theta<\pi$, there exists a positive number $t(\theta)$ such that for all $0<t \leq t(\theta)$, there exists a properly embedded minimal annulus $\widetilde{A}(\theta, t)$, which is not an unduloid or a helicoid, and which is the lift of a compact minimal torus in $(S \times \mathbb{R}) / \tau(\theta, t)$, where $\tau(\theta, t)$ is the screw motion symmetry which acts on $S$ by rotation around the $x_{3}$-axis by angle $2 \theta$ and on $\mathbb{R}$ by translation by $2 t$. Furthermore, 
$\widetilde{A}(\theta, t)$ is invariant under reflection across a vertical annulus of symmetry and under reflection across a great circle in $S \times\left\{\frac{t}{2}\right\}$.

It turns out that Lemma 2.1 and Theorem 2.1 hold for other metrics on a sphere $S^{2}$. We briefly explain this construction for these metrics.

Assume that $S^{2}$ is equipped with a metric invariant under an $S^{1}$ action with two fixed points $p, q \in S^{2}$. Let $\gamma_{p, q}$ be a least length geodesic arc on $S^{2}$ joining $p$ to $q$ and let $\gamma_{p, q}^{\prime}$ be the rotation $R_{\pi}$ of $\gamma_{p, q}$ by angle $\pi$. Identify $S^{2}$ with $S^{2} \times\{0\}$ and consider the simple closed geodesic $\gamma=\gamma_{p, q} \cup \gamma_{p, q}^{\prime}$. Let $G$ be the associated $S^{1}$ action on $S^{2} \times \mathbb{R}$ and note that $G$ is a subgroup of the larger group $G \times \mathbb{R}$ of isometries of $S^{2} \times \mathbb{R}$, where $\mathbb{R}$ acts on $S^{2} \times \mathbb{R}$ by vertical translation. It is straightforward to check the orbit $S_{\theta}(\gamma)$, under any of the one-parameter screw motion subgroups $S_{\theta}$ in $G \times \mathbb{R}$, gives rise to a minimal "helicoid" in $S^{2} \times \mathbb{R}$ foliated by geodesics. Also note that the helicoid $S_{\theta}(\gamma)$ is invariant under the isometry which is the composition of $(x, t) \mapsto\left(R_{\pi}(x),-t\right)$, which one can consider to be rotation around $\gamma$. Similarly, $S_{\theta}(\gamma)$ is invariant under rotation around any of its horizontal geodesics. With little change, the proofs of Lemma 2.1 and Theorem 2.1 generalize to produce a related one-parameter family of embedded minimal annuli. If, in addition, one assumes that the metric on $S^{2}$ is invariant under a reflectional symmetry that interchanges $p$ and $q$, then the annuli produced by this method can be chosen to have the related reflectional symmetry in $S^{2} \times \mathbb{R}$.

We now give the proof of Lemma 2.1.

Proof. Let $p(\theta, t)=\left(\cos \left(\frac{-\theta}{2}\right), \sin \left(\frac{-\theta}{2}\right), 0\right)$. Fix $\theta$ and assume that $t$ is chosen so that $W(\theta, t)$ is stable. Let $D$ denote the disk on $S \times\{0\}$ which bounds $\alpha(0)$ and is disjoint from $p(\theta, 0)$ and let $E$ be the disk on $S \times\{t\}$ which bounds $\alpha(t, \theta)$ and is disjoint from $p(\theta, t)$. Note that there exists a one-parameter family of embedded annuli in $S \times[0, t]-W(\theta, t)$ with boundary $\alpha(0) \cup \alpha(\theta, t)$ which are topologically parallel to $W(\theta, t)$ and join the integral currents $W(\theta, t)$ and $D \cup E$; these annuli can also be chosen to have all the symmetries of $\alpha(0) \cup \alpha(\theta, t)$. Let $B$ be the ball in $S \times[0, t]$ bounded by $W(\theta, t) \cup D \cup E$. In $B$ the union $\alpha(0) \cup \alpha(\theta, t)$ bounds both the stable helicoid $W(\theta, t)$ and the stable surface $D \cup E$. As we just observed, these stable surfaces can be joined by a continuous family of embedded annuli with boundary $\alpha(0) \cup \alpha(\theta, t)$. A standard minimax argument (see, for example, [28] and [39]) implies that $\alpha(0) \cup \alpha(\theta, t)$ is the boundary of an index-one embedded minimal annulus $A(\theta, t) \subset S \times[0, t]-W(\theta, t)$. Since the deformation family of embedded annuli can be constructed to have the symmetries of $\alpha(0) \cup \alpha(\theta, t)$, the results in [39] imply $A(\theta, t)$ can be chosen to have the symmetries of $\alpha(0) \cup \alpha(\theta, t)$.

2.2. The two-parameter family $\mathcal{A}$ of embedded minimal annuli in $S \times \mathbb{R}$ foliated by circles. All of the minimal annuli described in the previous subsection are in 
fact in the family $\mathcal{A}$, which we will define and discuss here. We now describe the two-parameter family $\mathcal{A}$ of minimal annuli in $S \times \mathbb{R}$. These minimal annuli include the unduloids, the helicoids and their deformations, which we think of as Riemann type minimal surfaces [19] in $S \times \mathbb{R}$.

Constant mean curvature tori $M$ in $\mathbb{R}^{3}$ give rise to minimal annuli in $S \times \mathbb{R}$ as follows. The Gauss map $f$ of a constant mean curvature torus is a harmonic map to $S$. Its holomorphic Hopf quadratic differential is:

$$
Q(f)=\left[\left|\frac{\partial f}{\partial x}\right|^{2}-\left|\frac{\partial f}{\partial y}\right|^{2}-2 i\left\langle\frac{\partial f}{\partial x}, \frac{\partial f}{\partial y}\right\rangle\right] d z^{2},
$$

where $z=x+i y$ is a global holomorphic coordinate on the torus. Since $M$ is a torus, this is constant:

$$
Q(f)=c \cdot d z^{2}
$$

After a linear change of coordinates, we can assume that the constant $c$ is one. Then the map

$$
F: S^{1} \times \mathbb{R} \rightarrow S \times \mathbb{R}, \quad F(x, y)=(f(x, y), y)
$$

is a conformal harmonic map, i.e., a minimal surface. The Delaunay surfaces ( $f$ is periodic, as defined on a torus) yield the unduloids in $S \times \mathbb{R}$ and the nodoids yield the helicoids in $S \times \mathbb{R}$ under this correspondence by $F$.

Abresch studied the family of Wente tori (constant mean curvature $\frac{1}{2}$ in $\mathbb{R}^{3}$ ), where one family of principal curvature lines are planar [1]. More generally, he studied constant mean curvature surfaces parametrized by $\mathbb{R}^{2}$, with the coordinate axes $x$ and $y$ yielding the (smaller and larger) principal lines of curvature.

Those tori whose small curvature lines, $\lambda_{1}$, are planar were found among the solutions of the system:

$$
\begin{gathered}
\Delta \omega+\sinh (\omega) \cdot \cosh (\omega)=0 \\
\sinh (\omega) \cdot \dot{\omega}^{\prime}-\cosh (\omega) \cdot \dot{\omega} \omega^{\prime}=0 .
\end{gathered}
$$

Here' and denote the derivatives with respect to $x$ and $y$, respectively. We remark that $Q(f)=d z^{2}$ for these surfaces.

Abresch classified all real analytic solutions $\omega: \mathbb{R}^{2} \rightarrow \mathbb{R}$ of the above system in terms of elliptic functions. He then went on to study the system which corresponds to the larger curvature lines $\lambda_{2}$ being planar curves. It follows that the Gaussian image of each such $\lambda_{2}$ line is contained in a circle of $S$. These examples are solutions of the following system (I):

$$
\begin{gathered}
\Delta \omega+\sinh (\omega) \cdot \cosh (\omega)=0 \\
\cosh (\omega) \cdot \dot{\omega}^{\prime}-\sinh (\omega) \cdot \dot{\omega} \omega^{\prime}=0 .
\end{gathered}
$$


We remark that $Q(f)=-d z^{2}$ when $f$ is the Gauss map of this Abresch family. Abresch classifies all solutions of this system as well (it is analogous to the first solution space), and shows that the associated constant mean curvature surfaces do not close up in $\mathbb{R}^{3}$, and so, do not yield Wente tori. However, the solutions of system (I) do yield minimal immersions $F: S^{1} \times \mathbb{R} \rightarrow S \times \mathbb{R}$. Laurent Hauswirth [13] observed that the second equation of system (I) is precisely the condition that the level curves of $F$ be circles. We define $\mathcal{A}$ to be the family of these minimal surfaces, induced by solutions of (I). If $f$ denotes the Gauss map of a constant mean curvature surface solution of (I), then the minimal surface $(x, y) \mapsto(f(x, y), x)$ in $S \times \mathbb{R}$ is foliated by circles in level set spheres. We now discuss these surfaces from another point of view.

2.3. The existence of minimal annuli in $\widetilde{M} \times \mathbb{R}$, foliated by circles in a space form $\widetilde{\boldsymbol{M}}$. Hauswirth [13] observed that the classical Shiffman Jacobi function (defined below) for minimal surfaces in $\mathbb{R}^{3}$ (transverse to the planes $\mathbb{R}^{2} \times\{t\}$ ) generalizes to $\widetilde{M} \times \mathbb{R}$, where $\widetilde{M}$ is a space form of constant curvature. Shiffman [44] used this Jacobi function to prove that a compact minimal annulus in $\mathbb{R}^{3}$ with boundary circles in horizontal planes is foliated by circles in horizontal planes; hence, by Riemann [40], the compact annulus is contained in a periodic minimal surface of genus zero defined in terms of elliptic functions. This same argument works to prove that our examples in the previous subsection are foliated by circles, using the fact that the basic compact annular pieces are bounded by circles and have index of stability one. This technique also yields another way to generate Riemann type examples of minimal surfaces in $\widetilde{M} \times \mathbb{R}$, which we now describe.

Let $A$ be a compact minimal annulus in $\widetilde{M} \times \mathbb{R}$ with boundary curves in $\tilde{M} \times\left\{t_{1}\right\}$ and $\widetilde{M} \times\left\{t_{2}\right\}$ with $t_{1}<t_{2}$. Let $S(r)$ denote the circle of circumference $r$. Assume $S(r) \times\left[t_{1}, t_{2}\right]$ is a conformal parametrization of $A$ with $h(x, t)=t$ and where $r$ is the flux of $h$. Let $\kappa(\theta, t)$ be the corresponding geodesic curvature function of the level set curve at height $t$. Then,

$$
S=\lambda \frac{\partial \kappa}{\partial \theta}
$$

is a Jacobi function on $A$, where $\lambda(\theta, t)$ is the conformal factor or speed of the level set curve at the parameter values $(\theta, t)$. If the boundary curves of $A$ are chosen to be circles and $A$ is strictly stable, then the Shiffman function has zero boundary values on $A$, and so, vanishes on $A$. This means that $A$ is foliated by circles. In the case $\widetilde{M}$ is $S$, then, by analytic continuation, we obtain a periodic minimal surface $\widetilde{A}$. In fact, we obtain all the examples in $\mathcal{A}$ in this way. In the case $\widetilde{M}=\mathbb{R}^{2}$, we obtain a $\widetilde{A}$ which is a catenoid or one of the Riemann examples.

Let $\mathbb{H}$ be the hyperbolic plane. Now suppose one considers a small stable part of a catenoid in $\mathbb{H} \times \mathbb{R}$ bounded by a circle in $\mathbb{H} \times\{0\}$ and a circle in $\mathbb{H} \times\{t\}$. Translate sideways slightly the circle in $\mathbb{H} \times\{t\}$. Then, there is a new annulus $A(t, s)$ 
bounded by the two new circles and, using the Shiffman function, we see that it is foliated by circles. Notice that $A(t, s)$ has a vertical plane of symmetry coming from the symmetry of its boundary circles. Now $A(t, s)$ can be extended to a minimal surface $B(t, s)$ in some open neighborhood of $A(t, s)$ since $\partial A(t, s)$ is analytic. Clearly, $B(t, s)$ is also foliated by circles. Thus, there is a maximal open minimal annulus $\widetilde{A}(s)$ foliated by circles, and containing $A(t, s)$. A simple maximum principle argument (using complete catenoids as barriers) shows the asymptotic boundary of $\widetilde{A}(s)$ is non-empty. Hence, $\partial \widetilde{A}(s)$ consists of two horocycles $C_{1}$ and $C_{2}$. Assuming $C_{2}$ higher than $C_{1}, \widetilde{A}(s)$ extends above $C_{2}$ by level curves of constant curvature less than one, i.e., equidistant curves. These curves eventually become a geodesic $\gamma_{2}$. Similarly, extending $\widetilde{A}(s)$ below $C_{1}$ by equidistant curves, we arrive at a geodesic $\gamma_{1}$. Now rotate about $\gamma_{1}$, and $\gamma_{2}$ and continue to obtain a complete embedded Riemann type minimal surface in $\mathbb{H} \times \mathbb{R}$.

2.4. Connected sum construction. We now present a simple connected sum construction for creating properly embedded minimal surfaces of finite topology from old ones. Suppose $\Sigma$ is a properly embedded minimal surface of finite topology in $M_{1} \times \mathbb{R}$ and such that there exists a disk $D_{1} \subset M_{1}$ such that $\left(D_{1} \times \mathbb{R}\right) \cap \Sigma=\varnothing$. First take the connected sum of $M_{1}$ with another surface $M_{2} ; M_{1} \# M_{2}$ equals the disjoint union of $M_{1}-D_{1}$ and $M_{2}-D_{2}$ glued together along their boundary curves where $D_{2}$ is a disk in $M_{2}$. Next extend the Riemannian metric on $M_{1}-D_{1} \subset M_{1} \# M_{2}$ to all of $M_{1} \# M_{2}$; then we can view $\Sigma$ as being a minimal surface in $\left(M_{1} \# M_{2}\right) \times \mathbb{R}$. For example, consider a Scherk doubly-periodic surface of genus zero $\Sigma \subset \mathbb{T} \times \mathbb{R}$. Then, using this connected sum construction, we can consider $\Sigma$ to be a minimal surface in $(\mathbb{T} \# \mathbb{T}) \times \mathbb{R}$ with a metric of non-positive curvature on $\mathbb{T} \# \mathbb{T}$.

2.5. Construction of minimal annular graphs in $\boldsymbol{M} \times \mathbb{R}$. We now describe a method for constructing a connected annular minimal graph $G$ in $M \times \mathbb{R}$. By the Stability Theorem (Theorem 1.2 stated in the Introduction), any such graph $G$ must have a finite number of top ends which are asymptotic to the top ends of $\Gamma_{T} \times \mathbb{R}$, where $\Gamma_{T}=\{\alpha(1), \ldots, \alpha(T)\}$ is a finite collection of pairwise disjoint stable simple closed geodesics on $M$. Let $\Gamma_{B}=\{\beta(1), \ldots, \beta(B)\}$ be the family of geodesics corresponding to the bottom ends of $G$. Since the total flux of the top ends of $G$ is equal to the total flux of the bottom ends of $G$, then

$$
\sum_{n=1}^{T} l(\alpha(n))=\sum_{n=1}^{B} l(\beta(n)),
$$

where $l$ refers to the length of the geodesic. It follows from this formula that for an $M$ with a generic metric every minimal graph in $M \times \mathbb{R}$ must have some top end and some bottom end asymptotic to the same $\gamma \times \mathbb{R}$, since for a generic metric one 
does not have such a rational relationship for the lengths of distinct closed geodesics. However, for some special metrics on $M$ it is possible to construct such graphs where the geodesics in $\Gamma_{T}$ are disjoint from the geodesics in $\Gamma_{B}$. We now make one such construction.

Consider any Riemannian metric on the topological annulus $S^{1} \times[-1,1]$ that is invariant under the reflection $R,(p, t) \mapsto(p,-t)$, and such that there exists a least length simple closed geodesic $\alpha$ in $S^{1} \times[-1,1]$ with the length of $\alpha$ less than the length of $S^{1} \times\{0\}$. Suppose $\alpha$ is also chosen to be such a geodesic such that $\alpha$ is closest to $S^{1} \times\{0\}$. Since $R(\alpha)$ is disjoint from $\alpha, \alpha$ and $R(\alpha)$ bound a new annulus $A \subset S^{1} \times[-1,1]$. Consider the curves $\alpha(t)=\alpha \times\{t\}$ and $\beta(t)=R(\alpha) \times\{-t\}$. Let $\Sigma(t)$ be the area-minimizing surface in $A \times[-t, t]$ with boundary $\alpha(t) \cup \beta(t)$. A simple application of the maximum principle (Rado's theorem) implies that $\Sigma(t)$ is the unique compact minimal surface in $A \times[-t, t]$ with boundary $\Sigma(t)$ and that $\Sigma(t)$ is a graph over the annulus $A$. Since $\Sigma(t)$ is invariant under the isometry $(p, s) \mapsto(R(p),-s), \Sigma(t) \cap(A \times\{0\})=S^{1} \times\{0\}$. It now follows rather easily, using that $S^{1} \times\{0\}$ is longer than $\alpha$ and that $\Sigma(t)$ is area-minimizing, that the graphs $\Sigma(t)$ converge to a graph $G$ over $A$ with top end asymptotic to $\alpha \times[0, \infty)$ and bottom end asymptotic to $R(\alpha) \times(-\infty, 0]$.

The general importance of the example discussed in the previous paragraph is that it guarantees the existence of minimal annular graphs in those $M \times \mathbb{R}$ where such a metric on $S^{1} \times[-1,1]$ isometrically embeds in $M$. Therefore, a simple consequence of this result is the following theorem.

Theorem 2.2. Suppose $R: M \rightarrow M$ is an orientation reversing isometry of an orientable surface of positive genus with a fixed point component which is a strictly unstable geodesic. Then $M \times \mathbb{R}$ admits an annular minimal graph.

2.6. Existence of non-isotopic minimal graphs in $M \times \mathbb{R}$. As we observed in the previous subsection, for a generic metric on $M$, there do not exist connected minimal graphs where the multiplicity of the geodesics corresponding to the annular ends of the graphs is one. In the case $M$ has genus zero, this presents a genuine obstruction to finding minimal graphs. Also, if $M$ is a torus with a flat metric, then there do not exist any minimal graphs. However, for any compact orientable Riemannian surface $M$ of genus greater than one, there exist infinitely many minimal graphs in $M \times \mathbb{R}$, where some closed geodesic corresponding to a top end also corresponds to a bottom end. For example, for a generic metric on $M$, there exists a unique embedded closed geodesic $\Gamma$ of least length which does not separate $M$. For such a Riemannian surface, there exists a complete minimal graph $G$ over $M-\Gamma$ that is unique up to vertical translation (see the proof of the next theorem).

Theorem 2.3. If $M$ is orientable of genus at least one and $M$ is not a torus endowed with a metric which admits an infinite collection of distinct foliations by closed 
geodesics, then there exists an infinite number of non-isotopic domains in $M$ bounded by geodesics which minimize length in their homotopy class and such that there exist proper minimal graphs over these domains.

Proof. We will apply the existence theorem, Theorem 4.1 in Section 4, to obtain our graphs. The reader should consult Section 4 to see the definition of $M(\alpha, r) \subset$ $M \times S(r)$, where $S(r)$ is the circle of circumference $r$ and $\alpha \in H_{1}(M)$ is a primitive (non-multiple) homology class. We recall the essentials on the construction of these interesting surfaces. The homology class $\alpha$ can be represented by a simple closed curve $\bar{\alpha}$ with a product annular regular neighborhood $A=\bar{\alpha} \times[-\varepsilon, \varepsilon] \subset M$ with boundary circles $\alpha_{-\varepsilon}, \alpha_{\varepsilon}$. Consider $M$ to be the level set surface $M \times\{0\}$ for angle $\theta=0 \in S(r)$; here 0 denotes the identity element in $S(r)=\mathbb{R} / r \mathbb{Z}$. Next consider the annulus $G \subset M \times S(r)$ which is a linear graph of slope $\frac{r}{2 \varepsilon}$ over $A$ in $M \times S(r)$, where we consider the circle $S(r)$ to be a vertical line segment of height $r$ with the top and bottom points identified. A piecewise smooth surface $\widehat{M}(\alpha, r)$ is obtained by replacing the annulus $A$ on $M \times\{0\}$ by $G$. The surface $M(\alpha, r)$ is then an embedded surface of least-area in the homotopy class of this surface in $M \times S(r)\}$, which by Theorem 4.1 is unique up to translation. See Figure 1 for an indication of the construction of $\widehat{M}(\alpha, r)$.

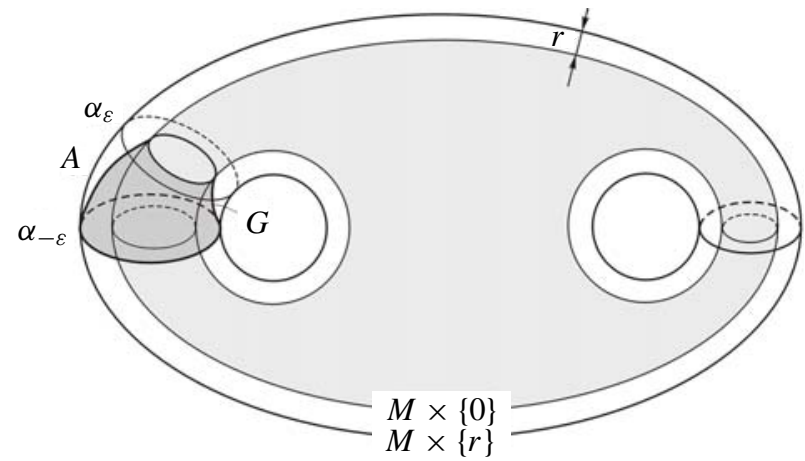

Figure 1. The surface $\widehat{M}(\alpha, r)$, where $M$ has genus two.

The minimal surface $M(\alpha, r)$ in $M \times S(r)$ is a graph over $M \times\{0\}$. Assume for the moment that the genus of $M$ is greater than one. In this case, we may assume, after a possible translation in $M \times S(r)$, that $M(\alpha, r)$ has a horizontal tangent space at height 0 (since the foliation of $M(\alpha, r)$ induced by intersecting with the $M \times\{t\}$ must have a singularity). Let $\mathcal{F}(r)$ be the minimal foliation of $M \times \mathbb{R}$ obtained from translates of lifts $\tilde{M}(\alpha, r)$ of $M(\alpha, r)$ to $M \times \mathbb{R}$. By letting $r_{i} \rightarrow \infty$ and taking a limiting subsequence, we may assume that $\widetilde{M}\left(\alpha, r_{i}\right)$ converges to an area minimizing surface $\Sigma(1)$ which is a leaf of a limit foliation $\mathcal{F}$ of the $\mathcal{F}\left(r_{i}\right)$. Note that 
$\mathcal{F}\left(r_{i}\right)$ is invariant under vertical translation and so is its limit $\mathcal{F}$. Since the fluxes of the $M\left(\alpha, r_{i}\right)$ are bounded from below by the flux of $M\left(\alpha, r_{1}\right)$ (see Proposition 4.1) and there is a uniform curvature estimate for stable minimal surfaces in $M \times \mathbb{R}$, Theorem 1.1 implies that every leaf of $\mathcal{F}$ is properly embedded. Since $r_{i} \rightarrow \infty$, Proposition 4.1 implies that the leaves of $\mathcal{F}$ are not periodic, and so, by the Stability Theorem (Theorem 1.2), any component of $\Sigma(1)$ containing a nonvertical tangent space at some point is a graph over its projection to $M \times\{0\}$. The Stability Theorem also implies that any leaf of $\mathcal{F}$ with some vertical tangent space is a vertical annulus over a stable embedded geodesic in $M \times\{0\}$. By our choice of translation of $M(\alpha, r)$, $\Sigma(1)$ has a horizontal tangent space at some point in $M \times\{0\}$, and so, the component of $\Sigma(1)$ containing this point must be a graph.

Now let $C(1)$ be the union of the components of $\Sigma(1)$ containing critical points of $h$. Since $h: C(1) \rightarrow \mathbb{R}$ has at least one critical point in each component, $C(1)$ is a graph over a subdomain $\Delta(C(1)) \subset M \times\{0\}$ with each component of $\Delta(C(1))$ having negative Euler characteristic. If $\chi(\Delta(C(1)) \neq 2 g-2$, then, after different translations of $M\left(\alpha, r_{i}\right)$ in $M \times S\left(r_{i}\right)$, we can obtain a new limit surface $\Sigma(2)$ with $h: \Sigma(2) \rightarrow \mathbb{R}$ having at least one critical point and such that for the new set $C(2), \Delta(C(2))$ is disjoint from $\Delta(C(1))$. Continuing in this manner, we eventually obtain disjoint domains $\Delta(C(1)), \Delta(C(2)), \ldots, \Delta(C(n))$ in $M \times\{0\}$, such that every component of these domains has negative Euler characteristic and such that

$$
M \times\{0\}-\bigcup_{i=1}^{n} \Delta(C(i))
$$

consists of components which are compact annuli or which are stable simple closed geodesics. By construction, the primitive homology class $\alpha$ can be represented by a finite sum of the boundary curves of the domains $\Delta(C(i))$, where each boundary curve is oriented and appears at most once in this sum for each such domain. It follows that such a decomposition $\Delta(C(1)), \ldots, \Delta(C(n))$ of $M \times\{0\}$, defined up to isotopy in $M \times\{0\}$, can only give rise to a finite number of primitive homology classes, since there are only a finite number of combinations of the boundary curves. Since there are an infinite number of primitive homology classes in $H_{1}(M \times\{0\})$, there exists an infinite number of non-isotopic domains in $M$ bounded by stable geodesics, which admit proper minimal graphs asymptotic to ends of flat annuli over these stable geodesics. This proves the theorem in the case the genus of $M$ is greater than one.

Now assume that $M$ has genus one. Let $\alpha$ be a primitive homology class in $H_{1}(M)$ such that $M$ is not foliated by simple closed geodesics that represent $\alpha$. As before, we have associated foliations $\mathcal{F}\left(r_{i}\right)$ and a limit foliation $\mathcal{F}$. As before, $\mathcal{F}$ consists of leaves which are graphs over annuli or which are vertical annuli over simple closed geodesics in $M \times\{0\}$. It also follows that for any leaf of $\mathcal{F}$ which is a graph over an annulus on $M$, each of its boundary curves represents $\alpha$. Thus, since we are assuming 
that $M \times\{0\}$ cannot be foliated by simple closed geodesics which represent $\alpha$, then $\mathcal{F}$ contains a leaf which is an annular graph over an annulus $\Delta(\alpha) \subset M \times\{0\}$. Since $H_{1}(M)$ has an infinite number of different primitive elements, $M$ must have an infinite number of non-isotopic annular domains which admit proper minimal graphs. This completes the proof of Theorem 2.3.

2.7. A heuristic approach for constructing Scherk towers. We now consider a possible final method for constructing minimal surfaces of finite topology in a general $M \times \mathbb{R}$; this proposed method is based on the desingularization procedure developed by Kapouleas [14] to approximate two transversely intersecting properly embedded non-degenerate minimal surfaces in a Riemannian three-manifold. For example, suppose $\Gamma$ is a collection of $K$ strictly stable pairwise disjoint geodesics in $M$. Let $\Sigma$ be a finite collection of surfaces of the form $\Sigma=\left\{M \times\left\{t_{1}\right\}, \ldots, M \times\left\{t_{n}\right\}\right\}$. Then, for $k$ sufficiently large, the method of Kapouleas suggests that there exists a sequence of properly embedded minimal surfaces $\Sigma(k)$ of genus $k$ and with $2 K$ vertical annular ends such that as $k \rightarrow \infty, \Sigma(k)$ converges to $\Sigma \cup(\Gamma \times \mathbb{R})$. The construction of $\Sigma(k)$ first entails replacing a small neighborhood of $\Gamma$ in $\Sigma \cap(\Gamma \times \mathbb{R})$ by a Scherk necklace, and then producing a small graph over this surface which is minimal. These $\Sigma(k)$ would then have index of stability approximately equal to $k$. Another variant of this heuristic method is to assume that $\Sigma$ is empty but allow $\Gamma$ to contain intersecting embedded geodesics and then desingularize $\Gamma \times \mathbb{R}$ by periodic properly embedded minimal surfaces, in much the same way that Scherk's singlyperiodic minimal surfaces desingularize two intersecting planes in $\mathbb{R}^{3}$. It would be nice to know if either of these heuristic methods can be made rigorous.

\section{The linear area growth theorem}

In this section, we will assume that $M$ is a compact orientable Riemannian surface of genus $g$ and that $\Sigma$ is a properly embedded orientable minimal surface with compact boundary and bounded curvature contained in $M \times \mathbb{R}$. Our goal is to prove that $\Sigma$ has linear area growth, from which it easily follows that the surface has a finite number of ends. An important tool for proving these results is the flux of $\Sigma$, which we now define.

Definition 3.1. Given a properly immersed minimal surface $\Sigma$ in $M \times \mathbb{R}$ without boundary, we define the flux of $\Sigma$ to be the flux of $\nabla h$ across $\Sigma \cap(M \times\{0\})$, where $h: \Sigma \rightarrow \mathbb{R}$ is the harmonic height function $h(p, t)=t$. Since $h$ is a proper harmonic function, the flux $F(\Sigma)$ of $\Sigma$ is the flux of $\nabla h$ across any level set of $h$, not just the level set at height zero.

Theorem 1.1 in the Introduction is a simple consequence of the statement and 
proof of the next theorem, which is the main result of this section.

Theorem 3.1. Suppose that $\Sigma$ is a connected properly embedded minimal surface with absolute curvature bounded by a constant $K_{0}$ in $M \times[0, \infty)$ and $\Sigma$ has compact boundary $\partial \Sigma \subset M \times\{0\}$. Then:

(1) $\Sigma$ has linear area growth in the sense that for every $\varepsilon>0$, there exist a constant $C(\varepsilon)$ such that the area of $\Sigma$ in $M \times[a, a+\varepsilon]$ is less than $C(\varepsilon)$ for all positive $a$. The constant $C(\varepsilon)$ depends on $M$, the flux $F(\Sigma)$ and the homology class of $[\partial \Sigma] \in H_{1}(M \times\{0\})$.

(2) If the tangent planes to $\Sigma$ make an angle of less than $\frac{\pi}{4}$ with the horizontal, then $\Sigma$ has one end and an end representative $E(\Sigma)$ such that the vertical projection $\pi: E(\Sigma) \rightarrow M \times\{0\}$ embeds as the end of a connected infinite cyclic covering space $\tilde{\pi}: \widetilde{M} \rightarrow M \times\{0\}$. In particular, $\partial \Sigma$ represents a non-zero primitive homology class in $H_{1}(M \times\{0\})$.

(3) There exists a positive constant $F_{M}$ depending only on $M$ such that if the flux $F(\Sigma) \leq F_{M}$, then the tangent planes to $\Sigma$ make an angle of less than $\frac{\pi}{4}$ with the horizontal. In particular, $\Sigma$ is stable when $F(\Sigma) \leq F_{M}$.

(4) For every positive $\varepsilon$ less than one and for every positive number $F$, there exists a positive number $A(\varepsilon, F)$ depending only on $M$ such that if the flux $F(\Sigma) \leq F$ and for some $a>1$, the area of $\Sigma \cap(M \times[a, a+\varepsilon])$ is greater than $A(\varepsilon, F)$, then the tangent planes to $\Sigma$ make an angle less than $\frac{\pi}{4}$ with the horizontal.

By the monotonicity formula for area, the area of a compact minimal surface with boundary in a ball in $M \times \mathbb{R}$ of radius $\varepsilon$ and centered at a point on the surface has area greater than $\varepsilon^{2}$ for $\varepsilon$ small and fixed. Since $M \times \mathbb{R}$ has two ends and each end representative of $\Sigma$ has area at least $\varepsilon^{2}$ in either $M \times[a, a+\varepsilon]$ or $M \times[-a,-a+\varepsilon]$ for $a$ large, then statement (1) of the above theorem implies that if $\Sigma$ has an infinite number of ends, then it does not have bounded curvature. Thus, the following corollary holds.

Corollary 3.1. If $\Sigma$ is a properly embedded minimal surface with bounded curvature and compact boundary in $M \times \mathbb{R}$, then $\Sigma$ has a finite number of ends.

We first prove a proposition concerning connected infinite cyclic covering spaces of $M$ that will be useful in the proof of Theorem 3.1, as well as in the proofs of some of our later theorems.

Proposition 3.1. Suppose $p_{1}: M_{1} \rightarrow M$ and $p_{2}: M_{2} \rightarrow M$ are two connected infinite cyclic covering spaces of a closed oriented surface $M$ of genus $g$. Then, these covering spaces are isomorphic in the sense that there exists a diffeomorphism $f: M \rightarrow M$ such that $f \circ p_{1}: M_{1} \rightarrow M$ lifts to $p_{2}: M_{2} \rightarrow M$. 
Proof. Since $p_{1}: M_{1} \rightarrow M$ is an infinite cyclic covering space, it corresponds to the kernel of a surjective representation $\sigma: \pi_{1}(M) \rightarrow \mathbb{Z}$. Since $\mathbb{Z}$ is abelian, $\sigma$ factors through the projection $\pi_{1}(M) \rightarrow \pi_{1}(M) / C=H_{1}(M)$, where $C$ is the commutator subgroup of $\pi_{1}(M)$ and where $H_{1}(M)$ is the first homology group of $M$. Let $\widehat{\sigma}: H_{1}(M) \rightarrow \mathbb{Z}$ be the representation induced by $\sigma$.

We now give a description of all such representations in terms of primitive (nonmultiple) classes in $H_{1}(M)$. Let $\beta$ be a primitive class in $H_{1}(M)$. Since $\beta$ is a primitive class, Theorem 1 in [18] implies that we can represent $\beta$ by a simple closed curve $\bar{\beta}$ on $M$. Consider the surface $M-\bar{\beta}$, and the associated compact oriented surface $M_{\bar{\beta}}$, which is the compact surface of genus $g-1$ obtained by attaching copies of $\bar{\beta}$ on each end of $M-\bar{\beta} . M_{\bar{\beta}}$ is oriented from the orientation induced from $M$. We label the boundary curves of $M_{\bar{\beta}}$ by $\left\{\bar{\beta}_{+}, \bar{\beta}_{-}\right\}$with $\bar{\beta}_{+}$being the boundary curve whose orientation agrees with the orientation of $\beta$ in $M$.

Since $H_{1}\left(M_{\bar{\beta}}\right)=\mathbb{Z}^{2 g-1}$ and under the inclusion map one can consider $H_{1}\left(M_{\bar{\beta}}\right)$ to be a subgroup of $H_{1}(M)$, we can consider $H_{1}(M)=\mathbb{Z} \oplus H_{1}\left(M_{\bar{\beta}}\right)$, where $(1,0)$ is represented by a simple closed curve $\alpha$ that intersects $\bar{\beta}$ transversely in a single point with +1 intersection number. Consider the surjective quotient homomorphism $\widehat{\sigma}_{\bar{\beta}}: H_{1}(M) \rightarrow \mathbb{Z}=H_{1}(M) / H_{1}\left(M_{\bar{\beta}}\right)$. This representation turns out to be independent of the choice of simple closed curve representative for $\beta$; in other words, we get a well-defined surjective homomorphism $\widehat{\sigma}_{\beta}: H_{1}(M) \rightarrow \mathbb{Z}$, which is just the primitive cohomology class in $H^{1}(M)$ corresponding to $\beta$ via Poincaré duality. Since the Poincaré dual of a primitive element in $H^{1}(M)$ is a primitive homology class, we see that every connected infinite cyclic covering space of $M$ can be realized by a $\widehat{\sigma}_{\beta}: H_{1}(M) \rightarrow \mathbb{Z}$ for some unique primitive class $\beta \in H_{1}(M)$.

Suppose that $\beta_{1}, \beta_{2}$ are two primitive homology classes represented by simple closed curves $\bar{\beta}_{1}, \bar{\beta}_{2}$, respectively. By the classification of surfaces, there exists an orientation preserving diffeomorphism $f: M_{\bar{\beta}_{1}} \rightarrow M_{\bar{\beta}_{2}}$ preserving the + and boundary curves and the identification map on each of the boundaries that give back the surface $M$. In particular, $f$ induces an orientation preserving diffeomorphism $\widehat{f}: M \rightarrow M$ such that $\widehat{f} \mid \bar{\beta}_{1}: \bar{\beta}_{1} \rightarrow \bar{\beta}_{2}$ in an orientation preserving manner and $\widehat{f}\left(M-\bar{\beta}_{1}\right)=M-\bar{\beta}_{2}$. By construction, $\widehat{f}_{*}\left(\operatorname{ker}\left(\widehat{\sigma}_{\beta_{1}}\right)\right)=\operatorname{ker}\left(\widehat{\sigma}_{\beta_{2}}\right)$. By elementary covering space theory, $\widehat{f}$ lifts to the associated covering spaces, and so, the covering spaces are isomorphic. This completes the proof of Proposition 3.1.

For our later applications, we will need a geometric picture of an infinite cyclic covering space that arises in the proof of Proposition 3.1 for a fixed primitive homology class $\beta \in H_{1}(M)$, which is represented by a simple closed curve $\bar{\beta}$. Let $M_{\bar{\beta}}$ be the compact surface defined in the proof of Proposition 3.1. For every $n \in \mathbb{Z}$ consider $M_{\bar{\beta}}(n)=M_{\bar{\beta}} \times\{n\}$ with associated boundary curves $\beta_{+}(n)$ and $\beta_{-}(n)$. Consider 
the space $\widetilde{M}_{\beta}=\bigcup_{n \in \mathbb{Z}} M_{\bar{\beta}}(n) / \sim$, where $\sim$ means that we identify points of $\beta_{+}(n)$ with the corresponding $\beta$ points on $\beta_{-}(n+1)$. Then, there is a natural projection $\pi_{\beta}: \tilde{M}_{\beta} \rightarrow M$, which is the infinite cyclic covering space of $M$ associated to the primitive homology class $\beta$. For later purposes, note that the curve $\beta_{+}(1)$ separates the two ends of $\widetilde{M}_{\beta}$. Also, note that if $\Gamma$ is a possibly disconnected finite collection of oriented simple closed curves that separate the two ends of $\widetilde{M}_{\beta}$ and are the oriented boundary of one of these ends, then, up to changing the orientation of $\Gamma, \Gamma$ is homologous to $\beta_{+}(1)$, and so, projects to a (possibly disconnected) representative for $\beta$. Further, note that $\mathbb{Z}$ acts naturally on $\widetilde{M}_{\beta}$ as a group of covering transformations, where the generator $\tau$ satisfies $\tau\left(M_{\bar{\beta}}(n)\right)=M_{\bar{\beta}}(n+1)$.

Proof of Theorem 3.1. For every $a \in(0, \infty)$, let $T_{a}: M \times[0, \infty) \rightarrow M \times \mathbb{R}$ be the translational isometry $T_{a}((p, t))=(p, t-a)$. Suppose now that $\Sigma$ fails to have linear area growth in $M \times[0, \infty)$. In this case there exists a sequence of $b_{n} \rightarrow \infty$ such that the area of $T_{b_{n}}(\Sigma)$ in $M \times\left[-\frac{1}{n}, \frac{1}{n}\right]$ is greater than $n$. We define $\Sigma(n)=T_{b_{n}}(\Sigma)$. Since the curvature of $\Sigma(n)$ in $M \times \mathbb{R}$ is bounded and $\Sigma$ is minimal and embedded, a subsequence of the $\Sigma(n)$ converges to a minimal lamination $\mathcal{L}$ of $M \times \mathbb{R}$ (see for example [27] or [38]).

We assert that $\mathcal{L}$ contains $M \times\{0\}$ as a leaf. Suppose for the moment that there is a leaf of $\mathcal{L}$ which intersects $M \times\{0\}$ and is not equal to $M \times\{0\}$. Every such leaf of $\mathscr{L}$ intersects $M \times\{0\}$ transversely at some point. Since the area of $\Sigma(n) \cap\left(M \times\left[-\frac{1}{n}, \frac{1}{n}\right]\right)$ goes to infinity, we may assume, after possibly going to a subsequence, that there exists a leaf $L$ of $\mathcal{L}$ which is either a limit leaf or has infinite area multiplicity as a limit of the $\Sigma(n)$. Furthermore, $L$ can be chosen so that there is a point $p \in L \cap(M \times\{0\})$ where the tangent plane to $L$ is not horizontal. For some small geodesic ball $B$ in $M \times \mathbb{R}$ centered at $p$ of radius $r$, the tangent planes to $\mathcal{L} \cap B$ make a positive angle of at least $\theta_{0}$ with the horizontal. The point $p$ can also be chosen so that, after choosing a subsequence, the area of $\Sigma(n)$ in $B$ is at least $n$ and the tangent planes to $\Sigma(n)$ in $B$ make an angle of at least $\theta_{0} / 2$ with the horizontal. It follows that the fluxes of the $\Sigma(n)$ across $M \times\{0\}$ are unbounded. But, the flux of $\Sigma(n)$ is equal to the flux of $\Sigma$, which gives a contradiction and thereby proves our assertion.

In the case $M$ is topologically a two-sphere, the proof of statement (1) of the theorem is now very simple and we explain it before proceeding. Note that a standard monodromy argument shows that when $M$ is simply connected, then the unbounded sheeting nearby $M \times\{0\}$ implies that one can lift $M \times(0)$ to the nearby leaves, so the $\Sigma(n)$ would be compact. This contradiction proves that if the area of $\Sigma$ in some region $M \times[a, a+\varepsilon]$ is sufficiently large, then the genus of $M$ is not zero.

Recall that we have shown that the surface $M \times\{0\}$ is one of the leaves of $\mathcal{L}$. Since $\Sigma(n)$ has bounded curvature, there exists an $\varepsilon>0$ such that every component of $\Delta(n)=\Sigma(n) \cap(M \times[-\varepsilon, \varepsilon])$ is almost horizontal for $n$ large. Furthermore, for $n$ large, there are a finite number $N(n)$ of components of $\Delta(n)$, each of which 
has boundary in both components of $\partial(M \times[-\varepsilon, \varepsilon])$ by the maximum principle for harmonic functions. Let $\Sigma(n, \varepsilon, 1), \Sigma(n, \varepsilon, 2), \ldots, \Sigma(n, \varepsilon, N(n))$ be a listing of the components of $\Delta(n)$.

The choice of $\varepsilon$ can be made to depend only on the bound $K_{0}$ for the curvature of $\Sigma$ and on the surface $M$. An effective version of this property is the following for $\varepsilon$ sufficiently small. Suppose $N$ is an embedded compact minimal surface in $M \times[-4 \varepsilon, 4 \varepsilon]$ with boundary in $M \times\{-4 \varepsilon, 4 \varepsilon\}$ with fixed flux and curvature bounded by $K_{0}$. If the area of $N \cap(M \times[-\varepsilon, \varepsilon])$ is sufficiently large (relative to its flux), then the angle that the tangent planes to $N$ make with the horizontal in $M \times[-2 \varepsilon, 2 \varepsilon]$ is less than $\frac{\pi}{4}$. From this point on in the proof of the theorem, we will assume that $\varepsilon$ is chosen small enough so that this horizontal property holds for such $N$. We note that this additional assumption on $\varepsilon$ does not affect the proof of the statement of Theorem 3.1. Also, note that the choice of $\frac{\pi}{4}$ is arbitrary and can be replaced by any small $\delta>0$, if one is willing to choose $\varepsilon$ sufficiently small and then assume that the area of $N$ is sufficiently large relative to its flux.

We summarize part of this discussion in the next assertion.

Assertion 3.1. Suppose $N$ is a compact embedded minimal surface in $M \times[-4 \varepsilon, 4 \varepsilon]$ with absolute curvature at most $K_{0}$ and $\partial N \subset M \times\{ \pm 4 \varepsilon\}$. For small $\varepsilon>0$, there exists an $N(\varepsilon)>0$ depending on the flux of $N$ such that if the area of $N \cap(M \times[-\varepsilon, \varepsilon])$ is at least $N(\varepsilon)$, then the tangent planes to $N \cap(M \times[-2 \varepsilon,+2 \varepsilon])$ make an angle of less than $\frac{\pi}{4}$ with the horizontal.

Let $F(\Sigma)$ be the flux of $\Sigma$, and define the number $F(n) \in(0, F(\Sigma)]$ to be the maximum of the fluxes of the components $\Sigma(n, \varepsilon, 1), \ldots, \Sigma(n, \varepsilon, N(n))$ across $M \times\{0\}$. Since the flux of $\Sigma$ is positive and equals the sum of the fluxes of $\Sigma(n, \varepsilon, 1), \ldots, \Sigma(n, \varepsilon, N(n))$, the first statement in Theorem 3.1 will follow by contradiction, once we show that the positive integers $N(n)$ are uniformly bounded and that $\lim _{n \rightarrow \infty} F(n)=0$.

In order to prove that $\lim _{n \rightarrow \infty} F(n)=0$, it suffices to prove that there exists a simple closed $C^{1}$-curve $\gamma$ on $M \times\{0\}$ such that for each $i, 1 \leq i \leq N(n)$, there exist simple closed $C^{1}$-curves $\gamma(n, i) \subset \Sigma(n, \varepsilon, i)$ which converge $C^{1}$ to $\gamma$ and such that $\gamma(n, i)$ is homologous to $\Sigma(n, \varepsilon, i) \cap(M \times\{\varepsilon\})$ in $\Sigma(n, \varepsilon, i)$. The reason that this suffices is that the lengths of the $\gamma(n, i)$ are then uniformly bounded. Hence, as $n \rightarrow \infty$, the $\gamma(n, i)$ are converging $C^{1}$ to $\gamma \subset M \times\{0\}$ and the tangent plane to $\Sigma(n)$ along $\gamma(n, i)$ is converging to the horizontal, and so, the flux across $\gamma(n, i)$ is converging to zero. By our homology condition, it follows that the flux of $\Sigma(n, \varepsilon, i)$ goes to zero as well. The proof of the boundedness of the $N(n)$ and the existence of the curves $\gamma(n, i)$ will occupy several pages.

For the remainder of the proof fix a collection $\mathscr{B}=\left\{\alpha_{1}, \beta_{1}, \ldots, \alpha_{g}, \beta_{g}\right\}$ of oriented simple closed curves on $M$ meeting at a single point, which generate a basis of $\pi_{1}(M)$. At times we will also consider $\mathscr{B}$ to lie in $M \times\{0\}$. 
We know that for large $n, \Sigma(n, \varepsilon, i)$ is a high order multigraph (with boundary) which submerses by the projection $\pi_{n}: \Sigma(n, \varepsilon, i) \rightarrow M \times\{0\}, \pi_{n}((p, t))=(p, 0)$. By embeddedness of $\Sigma$, given $\gamma \in \mathscr{B},(\gamma \times \mathbb{R}) \cap \Sigma(n, \varepsilon, i)$ either contains some components which are closed curves close to $M \times\{0\}$ or contains some components which are arcs close to $M \times\{0\}$ which spiral around $\gamma \subset M \times\{0\}$ and have end points on distinct components of $\partial(M \times[-\varepsilon, \varepsilon])$, but not both possibilities. Finally, note that since $\Sigma$ is non-compact, at least one of the basis curves for the fundamental group gives rise to spirals.

From this picture, we assert that one can construct an associated infinite cyclic covering space $\widetilde{\pi}_{n}: \widetilde{M} \rightarrow M \times\{0\}$. To see this, first consider the holonomy representation $\sigma_{n}$ on the standard basis $\mathscr{B}$ of $\pi_{1}\left(M, p_{0}\right)$ acting on the vertical fiber over some base point $p_{0} \in M \times\{0\}$, starting at some $\widetilde{p}_{0} \in \pi_{n}^{-1}\left(p_{0}\right)$ with $h\left(\widetilde{p}_{0}\right)$ close to zero. The fiber $\pi_{n}^{-1}\left(p_{0}\right)$ has a natural indexing by a finite interval of integers $I(n) \subset \mathbb{Z}$, where the ordering is by relative heights and with the index of $\tilde{p}_{0}$ corresponding to 0 . Since the surfaces $\Sigma(n, \varepsilon, i)$ are high sheeted and almost flat near $\widetilde{p}_{0}$, we may assume, after taking a subsequence, that $I(n) \subset I(n+k)$ for all positive integers $n$ and $k$ and that $\bigcup_{n \in \mathbb{N}} I(n)=\mathbb{Z}$. For $n$ large, the inclusion of the simply connected abstract geodesic completion $\bar{W}$ of the fundamental domain $W$ of $M \times\{0\}$ bounded by $\bigcup_{j=1}^{g} \alpha_{j} \cup \beta_{j}$, lifts to $\Sigma(n, \varepsilon, i)$ with base point $p_{0}$ lifting to $\widetilde{p}_{0}$. In particular, we can define for each $\alpha_{k}$ or $\beta_{k}$ an integer $\sigma_{n}\left(\alpha_{k}\right)$ or $\sigma_{n}\left(\beta_{k}\right)$ in $I(n) \subset \mathbb{Z}$. This map induces a homomorphism. Considering $\widetilde{p}_{0}$ to be the zero element in the image $\mathbb{Z}$ of $\sigma_{n}: \pi_{1}(M \times\{0\}) \rightarrow \mathbb{Z}$, let $\pi_{n}: \widetilde{M} \rightarrow M \times\{0\}$ be the covering space corresponding to the kernel of $\sigma$. Here, of course, $\widetilde{p}_{0}$ depends on $n$ and $i$ and we choose $\widetilde{p}_{0}$ so that as $n \rightarrow \infty$, the height of $\tilde{p}_{0}$ converges to zero.

We now make an important comment to help orient the reader on our basic strategy. This strategy is to show that the number of spirals in $\Sigma(n) \cap(\gamma \times[-\varepsilon, \infty]$, where $\gamma$ is one of the fixed basis curves for the fundamental group, is bounded independently of $n$. This result easily implies that the $N(n)$ are uniformly bounded and only a finite number of different infinite cyclic covering spaces $\tilde{\pi}_{n}$ can arise.

The components of $\Delta(n)$ are cyclically ordered in the following sense. Suppose $\widetilde{W}_{1}$ and $\widetilde{W}_{1}^{\prime}$ in $\Sigma(n, \varepsilon, 1)$ are two consecutive lifts of the fundamental region $W=$ $M \times\{0\}-\mathscr{B}$ with the second lift above the first. Then, we can order vertically, by their relative heights over $\widetilde{W}_{1}$, the other components of $\Delta(n)$, so that there exist unique lifts $\widetilde{W}_{2} \subset \Sigma(n, \varepsilon, 2), \ldots, \widetilde{W}_{N(n)} \subset \Sigma(n, \varepsilon, N(n))$ of $W$ to the subregion of $W \times \mathbb{R}$ between $\widetilde{W}_{1}$ and $\widetilde{W}_{1}^{\prime}$. By the holonomy representation discussed before, we see that this ordering is independent of the choice of the lift $\widetilde{W}_{1}$.

It is now clear that the holonomy representation giving rise to the associated infinite cyclic covering space is the same for all the $N(n)$ components in $\Delta(n)$.

We will now prove the statements (2), (3), (4) and then we establish statement (1). 
Proof of statement (2). Suppose that the tangent planes to $\Sigma$ make an angle of less than $\frac{\pi}{4}$ with the horizontal. We orient $\Sigma$ by the pull back of the orientation of $M$ under the vertical projection $\pi: \Sigma \rightarrow M \times\{0\}$.

We first observe that $\Sigma$ does not separate $M \times[0, \infty)$. To see this, recall that for some $\gamma \in \mathscr{B},(\gamma \times[0, \infty)) \cap \Sigma$ contains a positive finite number of infinite spirals beginning at $\partial \Sigma$. Since $\Sigma$ is oriented by its projection to $M$, the spirals in $(\gamma \times[0, \infty)) \cap \Sigma$ are oriented in a consistent manner, i.e., they are oriented by the oriented intersection $(\gamma \times[0, \infty)) \cap \Sigma$, which coincides with the orientation they inherit from the projection $\pi$. It follows that the signed intersection number of $\gamma$ with $\partial \Sigma$ in $M \times\{0\}$ is equal in absolute value to the non-zero number of these spirals. Thus, $\partial \Sigma$ does not separate $M \times\{0\}$, and so, $\Sigma$ does not separate $M \times[0, \infty)$.

Let $\gamma^{\prime}$ be a simple closed curve in $M \times\{0\}$ such that $\gamma^{\prime}$ intersects $\partial \Sigma$ transversely in a single point. Note that $\left(\gamma^{\prime} \times[0, \infty)\right) \cap \Sigma$ contains exactly one spiral component. On the other hand, each end of $\Sigma$ intersects $\gamma^{\prime} \times[0, \infty)$ by the $\frac{\pi}{4}$ hypothesis. Hence, $\Sigma$ has exactly one end.

We now check that $\Sigma$ has an end representative which embeds the infinite cyclic covering space $\tilde{\pi}: \bar{M} \rightarrow M \times\{0\}$, associated to the holonomy representation of the submersion $\pi: \Sigma \rightarrow M \times\{0\}$. This will complete the proof of statement (2).

Let $\beta$ be the homology class associated to $\widetilde{\pi}: \widetilde{M} \rightarrow M \times\{0\}$ given in the proof of Proposition 3.1. By the proof of this proposition, if $\bar{\beta}$ is a simple closed curve representative for $\beta$, then $\bar{\beta}$ lifts to $\tilde{M}$. Furthermore, any closed curve in $M-\bar{\beta}$ lies in the kernel of the representation and hence lifts to $\tilde{\pi}: \widetilde{M} \rightarrow M \times\{0\}$, where we consider $M-\bar{\beta}$ to lie in $M \times\{0\}$. Since $M-\bar{\beta}$ has diameter bounded by some positive constant $C$ and $\pi: \Sigma \rightarrow M \times\{0\}$ is a submersion that shrinks the lengths of tangent vectors by at most $\frac{1}{\sqrt{2}}$, we can lift $M-\bar{\beta}$ to $\Sigma$ if we begin the lift at any point of height at least $\sqrt{2} C$. From the discussion following the proof of Proposition 3.1, it is clear that the end $E$ of $\Sigma$ can be represented by the union of all the closure of these lifts and $E$ corresponds to an end of the infinite cyclic covering space $\tilde{\pi}: \widetilde{M} \rightarrow M \times\{0\}$. Statement (2) now follows.

Proof of statement (3). We now give the proof of statement (3) in Theorem 3.1. Note that since $\Sigma$ is a surface with curvature bounded by $K_{0}$, there exists a $\delta>0$ such that every point $p \in \Sigma$ with tangent plane $T_{p} \Sigma$ making an angle of at least $\frac{\pi}{4}$ with the horizontal has a small intrinsic $\delta$-neighborhood of area greater than some positive constant and on this neighborhood the tangent planes to $\Sigma$ make an angle of at least $\frac{\pi}{8}$ with the horizontal. Furthermore, the cross sectional length of the intersection of this neighborhood of $p$ with the level set surface $M \times\{h(p)\}$ has length bounded from below by another constant. In particular, the flux of such a surface $\Sigma$ is bounded from below by a constant that only depends on $M$ and $K_{0}$. This completes the proof of statement (3). 
Assertion 3.2. There is a bound on the number of components $N(n)$ and there are only a finite number of different possible representations $\sigma_{n}: \pi_{1}(M \times\{0\}) \rightarrow \mathbb{Z}$.

Proof. Assume to the contrary that there are an infinite number of different representations $\sigma_{n}$ or that the $N(n)$ are unbounded. After choosing a subsequence and a rearrangement of $\mathcal{B}$, we may assume that $\sigma_{n}\left(\left[\alpha_{1}\right]\right) \geq n$, or $\sigma_{n}\left(\left[\alpha_{1}\right]\right) \geq 1$ and $N(n) \geq n$.

Let $p_{n}: \Sigma(n) \cap M \times[-\varepsilon, \varepsilon] \rightarrow M \times\{0\}$ be the associated vertical projection. Let $E$ be a regular annular neighborhood of $\alpha_{1}$ in $M \times\{0\}$. Since $\sigma_{n}\left(\left[\alpha_{1}\right]\right) \geq$ $n$, or $\sigma_{n}\left(\left[\alpha_{1}\right]\right) \geq 1$ and $N(n) \geq n, p_{n}^{-1}(E)$ consists of at least $n$ spiraling strip components. The areas of the $\Sigma(n) \cap(M \times[-\varepsilon, \varepsilon])$ are unbounded as $n \rightarrow \infty$, since $p_{n}^{-1}(E) \cap M \times[-\varepsilon, \varepsilon]$ contains at least $n$ components each with area at least $\eta$, where $\eta$ depends only on $E$ and $\varepsilon$. By Assertion 3.1, this unbounded area property implies that the tangent planes to $\Sigma(n) \cap(M \times[-2 \varepsilon, 2 \varepsilon])$ make an angle of less than $\frac{\pi}{4}$ with the horizontal.

Thus, for some fixed $n$ sufficiently large, the tangent planes to $\Sigma(n) \cap(M \times$ $[-2 \varepsilon, 2 \varepsilon]$ make an angle of less than $\frac{\pi}{4}$ with the horizontal. Furthermore, for this large value of $n$, the number of spirals of $\Sigma(n) \cap\left(\alpha_{1} \times[-2 \varepsilon, 2 \varepsilon]\right)$ is the same as the number of spirals of $\Sigma(n) \cap\left(\alpha_{1} \times[-\varepsilon, \varepsilon]\right)$, which creates large area in the region $M \times[0,2 \varepsilon]$. So, we can apply the previous argument to conclude that the number of spirals in $\Sigma(n) \cap\left(\alpha_{1} \times[-\varepsilon, 3 \varepsilon]\right)$ is the same number. Continuing, we see that for all $a>0$, the number of spirals of $\Sigma(n) \cap\left(\alpha_{1} \times[a-\varepsilon, a+\varepsilon]\right)$ is constant. However, the number of these spirals is equal to $\sigma_{n^{\prime}}\left(\left[\alpha_{1}\right]\right) \cdot N\left(n^{\prime}\right)$ for some divergent sequence of values $a=a_{n^{\prime}}$, which diverges as $n^{\prime} \rightarrow \infty$ by our hypothesis in the first paragraph of this proof. This contradiction proves that there are only a finite number of different possible representations $\sigma_{n}$ and the numbers $N(n)$ are bounded.

By the above assertion, after choosing a subsequence, we can and will assume from this point on in the proof that the representation $\sigma_{n}=\sigma$, is independent of $n$.

With these preliminaries in place, we now show how to construct the curves $\gamma(i, n) \subset \Sigma(n, \varepsilon, i)$ which we discussed at the beginning of the proof and which converge smoothly to a curve $\gamma \subset M \times\{0\}$. Recall, from the proof of Proposition 3.1, the special curve $\bar{\beta}$ for a cyclic covering space $\tilde{\pi}: \widetilde{M} \rightarrow M$ with representation $\sigma: \pi_{1}(M) \rightarrow \mathbb{Z}$. The simple closed curve $\bar{\beta}$ is defined by having the property that the connected surface $M-\bar{\beta}$ lifts to $\tilde{M}$. Now choose $\gamma$ to be $\bar{\beta}$. Clearly, there are lifts $\gamma(n, i) \subset \Sigma(n, \varepsilon, i)$ of $\gamma$ converging smoothly to $\gamma$. It remains to prove that $\gamma(n, i)$ is homologous to $\Sigma(n, \varepsilon, i) \cap(M \times\{\varepsilon\})$.

To do this, we will show that $\gamma(n, i)$ separates $\Sigma(n, \varepsilon, i) \cap(M \times\{-\varepsilon\})$ from $\Sigma(n, \varepsilon, i) \cap(M \times\{\varepsilon\})$. Choose a lift $\widehat{M-\gamma}$ of $M-\gamma$ to $\Sigma(n, \varepsilon, i)$ whose closure is in the $\frac{\varepsilon}{10}$ neighborhood of $M \times\{0\}$. Note that $\widehat{M-\gamma}$ separates $(M-\gamma) \times[-\varepsilon, \varepsilon]$ into a top and a bottom component. From the cyclic covering space picture of $\Sigma(n, \varepsilon, i)$ 
near this lift, it is clear $\gamma(n, i) \subset \partial(\widehat{M-\gamma})$ separates $\Sigma(n, \varepsilon, i) \cap(M \times\{-\varepsilon\})$ from $\Sigma(n, \varepsilon, i) \cap(M \times\{\varepsilon\})$.

Now let $\Delta$ be the component of $\Sigma(n, \varepsilon, i)$ bounded by $\gamma(n, i)$ and $\Sigma(n, \varepsilon, i) \cap$ $(M \times\{\varepsilon\}) . \Delta$ gives the required homology. As remarked at the beginning of the proof of the theorem, this last result completes the proof that $\Sigma$ has linear area growth.

Proof of statement (4). Arguing by contradiction, assume that statement (4) fails to hold. Let $\Sigma(n)$ be a sequence of surfaces satisfying the hypothesis of the theorem where $F(\Sigma(n))<F$ and such that the area $A(n)$ of $\Delta(n)=\Sigma(n) \cap(M \times[a, a+\varepsilon])$ is greater than $n$, for some $a>1$, but some tangent plane of $\Sigma(n)$ makes an angle greater than $\frac{\pi}{4}$ with the horizontal. The proof of Assertion 3.2 implies that there exists a bound on the number $N(n)$ of components of $\Delta(n)$ and on the size of the set of associated holonomy representations for these components (we bound the number of spirals over a basis for the fundamental group). By statement (3), the flux of each $\Sigma(n)$ is bounded from below by a positive $F_{M}$. The proof that we just carried out to show that $\Sigma$ has linear area growth, then contradicts that $A(n)>n$ and $F\left(\Sigma(n) \geq F_{M}\right.$

Proof of statement (1). We have already shown that $\Sigma$ has linear area growth. Letting $F=F(\Sigma)$, statement (4) implies that if the area of $\Sigma$ is greater than $A(\varepsilon, F)$, then the tangent planes of $\Sigma$ make an angle of less than $\frac{\pi}{4}$ with the horizontal. Assume the area is greater than $A(\varepsilon, F)$. It follows from the proof of statement (2) that $\Sigma$ embeds as the end of an infinite cyclic cover of $M \times\{0\}$ with the projection map agreeing with the vertical projection of $\Sigma$ to $M \times\{0\}$ and $\Delta(a)=\Sigma \cap(M \times[a, a+\varepsilon])$ is connected.

Let $\gamma$ be a simple closed curve of least length which represents the primitive homology class $[\partial \Sigma] \in H_{1}(M)$. Let $\tilde{\gamma}$ denote the lift of $\gamma$ to $\Delta(a)$. Since the flux of $\nabla h$ across $\tilde{\gamma}$ equals $F(\Sigma)$, our previous arguments imply that there exists a bound $C(\varepsilon)$ for the area of $\Delta(a)$, which only depends on the length of $\gamma$, which in turn only depends on the homology class $[\partial \Sigma]$. This estimate completes the proof of the theorem.

\section{Stable minimal surfaces in $M \times \mathbb{R}$}

Some of the stable minimal surfaces that can arise in $M \times \mathbb{R}$ are actually periodic multigraphs, which just means they are lifts of compact embedded "graphical" minimal surfaces in $M \times S(r)$, where $S(r)$ is a circle with circumference $r$. The following is a description of the moduli spaces of these special minimal surfaces.

Theorem 4.1. Let $M$ be a compact orientable Riemannian surface of genus $g$. For each primitive homology class $\alpha \in H_{1}(M)$ and each $r \in \mathbb{R}^{+}$, there exists a compact embedded minimal surface $M(\alpha, r) \subset M \times S(r)$ of genus $g$ such that its preimage 
or "lift" $\widetilde{M}(\alpha, r)$ to $M \times \mathbb{R}$, together with the vertical projection to $M \times\{0\}$, is the oriented infinite cyclic covering space of $M$ associated to $\alpha$. Furthermore, all vertical translations of $M(\alpha, r)$ yield a product minimal foliation of $M \times S(r)$ and $M(\alpha, r)$ is the unique minimal surface in its homotopy class up to translation. Also, $M(\alpha, r)$ minimizes area in its integer homology class.

Proof. Let $0 \in S(r)=\mathbb{R} / r \mathbb{Z}$ denote the identity element in $S(r)$. For a primitive class $\alpha \in H_{1}(M \times\{0\})$, it is easy to construct an embedding $\widehat{M}(\alpha, r)$ of $M$ into $M \times S(r)$ which satisfies the following:

(1) $\widehat{M}(\alpha, r)$ is a graph over $M \times\{0\}$ under the natural projection $\pi: M \times S(r) \rightarrow$ $M \times\{0\}$.

(2) If $\beta$ is a simple closed curve with $\alpha \cap[\beta]=+1$, then the lift $\widetilde{\beta}$ of $\beta$ to $\widehat{M}(\alpha, r)$ represents the oriented class $([\beta], 1)$ in $H_{1}(M \times S(r))=H_{1}(M) \times H_{1}(S(r))$.

(3) $\widehat{M}(\alpha, r) \cap M \times\{0\}$ is a simple closed curve which represents the homology class $\alpha$.

For an indication of exactly how to construct $\widehat{M}(\alpha, r)$, see the proofs of Theorem 3.1 and Proposition 3.1 in Section 3, or perhaps the construction is most easily seen from the discussion at the beginning of the proof of Theorem 2.3 and the related Figure 1. Also, note that our $\alpha$ corresponds to the homology class in the choice of the curve $\bar{\beta}$ given in the proof of Proposition 3.1.

Let $M(\alpha, r)$ be a minimal surface of least-area in the homotopy or the isotopy class of $\widehat{M}(\alpha, r)$ in $M \times S(r)$. The existence of $M(\alpha, r)$ follows from the results in [8] or [28] and the fact that $\widehat{M}(\alpha, r)$ is an incompressible surface in $M \times S(r)$. By applying standard surface replacement arguments as first described by Meeks and Yau in [29], one sees that any two distinct such least-area surfaces in the homotopy class of $\widehat{M}(\alpha, r)$ are disjoint. Therefore, vertical translations of $M(\alpha, r)$ are disjoint from $M(\alpha, r)$, and so, one obtains a foliation of $M \times S(r)$ with leaves isometric to $M(\alpha, r)$ and which topologically is a product foliation.

It follows from the existence of this minimal foliation that $M(\alpha, r)$ is the unique minimal surface in $M \times S(r)$ in the homotopy class of $M(\alpha, r)$ up to translation. Otherwise, there would be another such minimal surface $\Delta \subset M \times S(r)$. Lift $\Delta$ to $\widetilde{\Delta}$ in the infinite cyclic covering space $\widetilde{M \times S(r)}$ of $M \times S(r)$ corresponding to the subgroup $\pi_{1}(M(\alpha, r))$ and lift the "product" minimal foliation to $\overline{M \times S}(r)$. Note that some of the minimal leaves of this foliation of $\widetilde{M \times S(r)}$ are disjoint from $\widetilde{\Delta}$. Since the minimal foliation of $\widetilde{M \times S}(r)$ consists of compact leaves of the form $\{L(t) \mid t \in \mathbb{R}\}$, there is a largest $t_{0}$ such that $L\left(t_{0}\right) \cap \widetilde{\Delta} \neq \varnothing$. The maximum principle for minimal surfaces now implies that $L\left(t_{0}\right)=\widetilde{\Delta}$, which proves our assertion that $\Delta$ is one of the translates of $M(\alpha, r)$ in $M \times S(r)$. 
A well-known application of the divergence theorem is that a compact leaf of a codimension-one oriented minimal foliation is area minimizing in its integer homology class. We sketch the argument for the sake of completeness. Let $N$ be the unit normal vector field to the minimal foliation and let $L$ be a compact leaf. Suppose $\Sigma$ is a cycle homologous to $L$ that is not a leaf of the foliation and $\omega$ is a chain with $\partial \omega=L-\Sigma$. Since the foliation is minimal, the divergence of $N$ is zero. So, applying the divergence theorem, one obtains that the flux of $N$ across $L$ equals the flux of $N$ across $\Sigma$. Since the flux across $L$ is its area and the flux of $N$ across $\Sigma$ is less than or equal to its area, we obtain the desired result. This completes the proof of Theorem 4.1.

A basic tool for proving the Stability Theorem in [26] is the following proposition, which implies that $\widetilde{M}\left(\alpha, r_{1}\right)$ and $\widetilde{M}\left(\alpha, r_{2}\right)$ can be differentiated by their fluxes when $r_{1} \neq r_{2}$. One reason that we include the proof of this proposition here, rather than in [26], is that we need it in the construction of stable minimal graphs in $M \times \mathbb{R}$ in Section 2 (see the proof of Theorem 2.3).

Proposition 4.1. Fix any primitive homology class $\alpha \in H_{1}(M)$. For every $r>0$, the surface $M(\alpha, r)$ has positive flux $F(\alpha, r)$. Furthermore, $F(\alpha, r)$ is a continuous strictly increasing function from $\mathbb{R}^{+}$to $\mathbb{R}^{+}$.

Proof. Since $\alpha$ is fixed, we will suppress the index $\alpha$ in $M(\alpha, r)$ and $F(\alpha, r)$. For a convergent sequence $r_{i} \rightarrow r_{0}$, consider the surfaces $M\left(r_{i}\right) \subset M \times S\left(r_{i}\right)$. Note that in a natural way the metrics in $S\left(r_{i}\right)$ converge to the metric on $S\left(r_{0}\right)$, and so, we can consider the surfaces $M\left(r_{i}\right)$ to be surfaces, not minimal, in $M \times S\left(r_{0}\right)$. Since each $M\left(r_{i}\right)$ minimizes its area in its homology class in the metric on $M \times S\left(r_{i}\right)$, a limit of these surfaces with bounded second fundamental form exists, which can be assumed to be a $C^{2}$-surface, and is again area-minimizing in its homology class. Hence, by the uniqueness statement in Theorem 4.1, the limit surface is $M\left(r_{0}\right)$ and since the convergence is $C^{1}, F\left(r_{i}\right) \rightarrow F\left(r_{0}\right)$. This proves that $F$ is continuous as a function of $r$. Next consider the sequence $r_{n}=r_{0}-\frac{r_{0}}{n}$, where $n$ is a positive integer. A straightforward argument, using the continuity of $F$, shows that if $F\left(r_{n}\right)<F\left(r_{0}\right)$ for all $n$ and for all $r_{0}$, then $F$ is strictly increasing. Since our argument does not depend on the value $r_{0}$, we will assume for concreteness that $r_{0}=1$ and $r_{n}=1-\frac{1}{n}$.

We first prove the theorem in the easier-to-visualize special case where $M$ is a torus $M=\mathbb{T}=S^{1} \times S^{1}=\mathbb{R}^{2} / \mathbb{Z}^{2}$ and where $\alpha$ is represented by $S^{1} \times\{0\}$; however, we do not assume that the metric on $\mathbb{T}$ is the flat metric. (On the other hand, if $\mathbb{T}$ has a flat metric, then the tori $\widehat{\mathbb{T}}(n)$ defined below are linear and the strictly increasing property of $F$ is clear; actually, it is this well-known fact which motivates the following proof.) In this case, consider the torus $\widehat{\mathbb{T}}(n) \subset \mathbb{T} \times \mathbb{R} / \mathbb{Z}$ that is the image of the map $f: \mathbb{R} \times \mathbb{R} \rightarrow \mathbb{R}^{2} / \mathbb{Z}^{2} \times \mathbb{R} / \mathbb{Z}$ defined by $f\left(\theta_{1}, \theta_{2}\right)=\left(\theta_{1}, n \theta_{2},(n-1) \theta_{2}\right)$. Let $\mathbb{T}(n)$ be the least-area torus in the homotopy class of $\widehat{\mathbb{T}}(n)$. Note that $\mathbb{T}(n)$ lifts to $M \times S(n-1)$ 
and this lift is equal to the inverse image $\widetilde{M}\left(r_{n}\right)$ of $M\left(r_{n}\right)$ in $M \times S(n-1)$ under the projection of the $\mathbb{Z}_{n}$-covering space $M \times S(n-1) \rightarrow M \times S\left(r_{n}\right)$; the reason for this is that these two surfaces are homotopic and $\widetilde{M}\left(r_{n}\right)$ is the unique minimal surface in its homotopy class. Since the curve on $M\left(r_{n}\right)$ for which we calculate flux lifts to $\widetilde{M}\left(r_{n}\right)$, the flux of $\mathbb{T}(n)$ is equal to the flux of $M\left(r_{n}\right)$ and it remains to prove that the flux of $\mathbb{T}(n)$ is less than the flux of $M(1)$. After a fixed vertical translation of $\mathbb{T}(n)$, we may assume that $M(1)$ and $\mathbb{T}(n)$ intersect transversely in a finite collection of simple closed oriented curves $\Gamma=M(1) \cap \mathbb{T}(n)$. The orientation on the components of $\Gamma$ comes from the oriented intersection $M(1) \cap \mathbb{T}(n)$, where $M(1)$ and $\mathbb{T}(n)$ are both oriented by the projection to $M \times\{0\}$ and $M \times \mathbb{R}$ is oriented by the orientation of $M \times\{0\}$ given by the upward pointing unit normal in the direction $\frac{\partial}{\partial t}$ and with the standard orientation on $\mathbb{R}$ also given by $\frac{\partial}{\partial t}$. Thought of as a integral chain, $\Gamma$ represents the class $\alpha$, since this is the case for the homotopic torus $\widehat{\mathbb{T}}(n)$ and the intersection pairing is well-defined on homology.

Recall that if $\sigma \subset \Sigma_{1} \cap \Sigma_{2}$ is an arc in the intersection of two oriented surfaces with almost complex structures $J_{1}, J_{2}$ in an oriented three-manifold, then $\sigma$ is oriented by a tangent vector $T(\sigma)$ such that $\left(J_{1}(T(\sigma)), J_{2}(T(\sigma)), T(\sigma)\right)$ represents the orientation of the ambient space. With this in mind, let $J_{1}$ be the almost complex structure on $M(1)$ and $J_{2}$ be the almost complex structure on $\mathbb{T}(n)$ induced as graphs over $M \times\{0\}$. Let $T(t)$ be the unit tangent vector field to $\Gamma(t)$. Then, with respect to our orientations,

$$
\left\langle J_{1}(T(t)), \frac{\partial}{\partial t}\right\rangle>\left\langle J_{2}(T(t)), \frac{\partial}{\partial t}\right\rangle
$$

where $\langle$,$\rangle is the metric on M \times \mathbb{R}$. By the divergence theorem, the flux of $\frac{\partial}{\partial t}$ across $\Gamma$ on $M(1), T(n)$, respectively, can be found by integrating the left, right hand sides, respectively, of the above inequality. Hence, the flux of $M(1)$ is greater than the flux of $\mathbb{T}(n)$. This completes the proof of the proposition in the case that $M$ is a surface of genus one and $\alpha$ is represented by $S^{1} \times\{0\}$.

Since any primitive homology class $\alpha$ in the first homology group of a surface $M=\mathbb{T}$ of genus one can be assumed to be the class represented by $S^{1} \times\{0\}$ (after composing with a diffeomorphism of $\mathbb{T}$ ), the proposition is proved in the genus one case. But, the initial construction that we carried out in the case of genus one can be easily adapted to the case where $M$ has higher genus. This discussion completes the proof of the proposition.

The following theorem is Theorem 3.1 in [26]. It is the key first step in the proof of the Stability Theorem in [26] and its proof takes up most of that paper. We will need the statement of this theorem in the next section.

Theorem 4.2 ([26]). Suppose $\Sigma$ is a non-compact orientable properly embedded stable minimal surface with compact boundary in $M \times \mathbb{R}$. Then, either every end 
of $\Sigma$ is asymptotic to an end of some "lift" or preimage $\widetilde{M}(\alpha, r)$ of a fixed $M(\alpha, r)$ described in Theorem 4.1 or some sequence of vertical translates of $\Sigma$ converges on compact subsets of $M \times \mathbb{R}$ to $\Gamma \times \mathbb{R}$, where $\Gamma$ is a finite collection of pairwise disjoint simple closed stable geodesics on $M$.

\section{A finite number of ends}

Recall that in Section 3, we proved that if $\Sigma$ has bounded curvature, then it has a finite number of ends. We will need this result and some of the techniques developed in Section 3 to prove the similar result holds, when we drop the bounded curvature hypothesis.

In this section, we will prove Theorem 1.3. Theorem 1.3 states that a properly embedded minimal surface $\Sigma$ in $M \times \mathbb{R}$ has a finite number of ends. This theorem is an immediate consequence of the following more general result, where $\Sigma$ is allowed to have compact boundary.

Theorem 5.1. If $\Sigma \subset M \times \mathbb{R}$ is a properly embedded minimal surface with compact boundary, then $\Sigma$ has a finite number of ends.

For the proof of Theorem 5.1, we will need the following proposition on certain geodesic laminations of surfaces. We give its proof at the end of this section.

Proposition 5.1. Suppose $\Gamma_{1} \subset \Gamma_{2} \subset \cdots \subset \Gamma_{n} \subset \cdots$, where $\Gamma_{n}$ is a finite collection of pairwise disjoint simple closed geodesics on a closed orientable Riemannian surface $M$ of genus $g$. Then, there is a bound on the lengths of the geodesics in $\Gamma=\bigcup_{i=1}^{\infty} \Gamma_{i}$. In particular, the closure $\bar{\Gamma}$ of $\Gamma$ is a geodesic lamination of $M$ consisting of closed geodesics with uniformly bounded lengths.

We now proceed with the proof of Theorem 5.1.

Proof. We may assume that $\Sigma$ is connected, is contained in $M \times[0, \infty)$ and $\partial \Sigma \subset$ $M \times\{0\}$. Assume that $\Sigma$ has an infinite number of ends. We will derive a contradiction. Note that, after possibly taking a four-sheeted cover of $M \times \mathbb{R}$ and lifting $\Sigma$ to this cover, we may assume that $\Sigma$ and $M$ are both orientable.

Since $\Sigma$ has an infinite number of ends, there exists a sequence $b_{n} \rightarrow \infty$ such that $M \times\left\{b_{n}\right\}$ intersects $\Sigma$ transversely in a finite number of simple closed curves and $\Sigma(n)=\Sigma \cap\left(M \times\left[b_{n}, \infty\right)\right)$ consists of $N(n)$ connected components $\Sigma(1, n), \ldots, \Sigma(N(n), n)$ where $N(n) \geq n+1$.

Assertion 5.1. $\Sigma$ can be chosen with an infinite number of ends and so that the surfaces $\Sigma(1, n), \ldots, \Sigma(N(n), n)$ are unstable for all $n$. 
Proof. First note that Corollary 1.3 implies that $\Sigma$ fails to have bounded curvature, and so, by curvature estimates [43], $\Sigma$ is unstable.

Suppose now that $\Sigma(i, n) \subset \Sigma(n)$ is stable for some $i$ and $n$. After reindexing, assume that $\Sigma(i, n)=\Sigma(1, n)$. In this case, Theorem 4.2 implies that either every end of $\Sigma(1, n)$ is asymptotic to the top end of some translate of $\widetilde{M}(\alpha, r)$ or there is a divergent sequence of translations of $\Sigma(1, n)$ that converge to $\Gamma \times \mathbb{R}$, where $\Gamma$ is a finite collection of simple closed stable geodesics on $M$. If every end of $\Sigma(1, n)$ is asymptotic to some translate of $\widetilde{M}(\alpha, r)$, then the proof of Theorem 4.2 implies that for all $k$, every end of a stable component $\Sigma(i, k)$ is also asymptotic to a translate of the same $\widetilde{M}(\alpha, r)$. Let $\varepsilon$ be either the length of the shortest simple closed stable geodesic in $M$ or the flux of $M(\alpha, r)$, depending on which of the two cases occurs for the end behavior of $\Sigma(1, n)$. In particular, the number of components of $\Sigma(n)$ which are stable is bounded by $[$ Flux $(\Sigma) / \varepsilon]$, where $[t]$ denotes the largest integer less than $t$.

Now choose an $n_{0}$ so that the number of stable components of $\Sigma\left(n_{0}\right)$ is maximal. Since $\Sigma$ has an infinite number of ends, there is some component of $\Sigma\left(n_{0}\right)$, say $\Sigma\left(1, n_{0}\right)$, which has an infinite number of ends. By our choice of $n_{0}$, every subend of $\Sigma\left(1, n_{0}\right)$ is unstable. Now replace $\Sigma$ by $\Sigma\left(1, n_{0}\right)$ translated downward by distance $b_{n_{0}}$ to obtain the surface with the desired properties. This completes the proof of Assertion 5.1.

Assume now that the conclusions of Assertion 5.1 hold for $\Sigma$ and consider the surfaces $\Sigma(1,1), \Sigma(2,1), \ldots, \Sigma(N(1), 1)$. Since $M \times\left\{b_{1}\right\}$ is minimal and $\Sigma(1)$ is minimal and locally separating in $M \times\left[b_{1}, \infty\right)$, the standard barrier minimization procedure described in [25] or [30] yields two properly embedded least-area orientable minimal surfaces $\Sigma(1,+)$ and $\Sigma(1,-)$, each with boundary equal to $\partial \Sigma(1)$. Here, the + sign refers to the surfaces that arise in minimization process that takes place on the side of $\Sigma$ where the oriented normal points and the - sign refers to the surfaces that arise in the minimization process on the other side. The way in which we obtain the surfaces is as follows. We first produce stable minimal surfaces $\Sigma(1,1,+)$ and $\Sigma(1,1,-)$ as least-area surfaces in $\left(M \times\left[b_{1}, \infty\right)\right)-\Sigma(1)$ with boundary $\partial \Sigma(1,1)$; this is done inductively component by component using old components as new barriers. Continuing inductively produces both $\Sigma(1,+)$ and $\Sigma(1,-)$. In this minimization process, a given component of $\Sigma(1,+)$ or $\Sigma(1,-)$ with the same boundary as a component of $\Sigma(1)$ intersects $\Sigma(1)$ only along its boundary, since every component of $\Sigma(n)$ is unstable. We remark that $\Sigma(1,1,+)$ or $\Sigma(1,1,-)$ may be compact (in fact, contained in $M \times\left\{b_{1}\right\}$ ); this will happen when $\partial \Sigma(1,1)$ bounds in $M \times\left\{b_{1}\right\}$. Notice that $\Sigma(1,1,+) \cup \Sigma(1,1,-)$ bounds a domain $R_{1} \subset M \times\left[b_{1}, \infty\right)$ that contains $\Sigma(1,1)$.

Once one has constructed the two properly embedded (disconnected) stable minimal surfaces $\Sigma(1,+)$ and $\Sigma(1,-)$, then, using $\Sigma(1,+) \cup \Sigma(1,-)$ and $\Sigma(2)$ as barri- 
ers, one can construct two properly embedded minimal surfaces $\Sigma(2,+)$ and $\Sigma(2,-)$, with boundary $\partial \Sigma(2)$ in the same manner as before. At this stage, we have produced $\Sigma(2,1,+)$ and $\Sigma(2,1,-)$ in $M \times\left[b_{2}, \infty\right)-[\Sigma(1) \cup \Sigma(1,1,+) \cup \Sigma(1,1,-) \cup$ $\Sigma(2,1) \cup \cdots \cup \Sigma(N(1), 1)]$ with boundary $\partial \Sigma(2,1)$. In this case, the components of $\Sigma(2, \pm)$ are disjoint from the components of $\Sigma(1, \pm)$. Continuing inductively, one obtains the stable surfaces $\Sigma(1,+), \Sigma(1,-), \ldots, \Sigma(n,+), \Sigma(n,-), \ldots$, whose interiors are disjoint from one another.

For $c>0$, let $T_{c}(p, t)$ denote the downward translation $(p, t) \mapsto(p, t-c)$. By Theorem 4.2, there are two possible asymptotic structures for $\Sigma(n,+) \cup \Sigma(n,-)$; either all of the ends are asymptotic to translates of some $\widetilde{M}(\alpha, r)$ where $\alpha \in H_{1}(M)$ and $r$ are both fixed, or under some vertical translations $T_{c(n, i)}$, the $T_{c(n, i)}(\Sigma(n,+) \cup$ $\Sigma(n,-))$ converge as $i \rightarrow \infty$ to $\Gamma \times \mathbb{R}$, where $\Gamma$ is a finite collection of pairwise disjoint stable simple closed geodesics in $M$. It follows that there exists a positive increasing function $f: \mathbb{N} \rightarrow \mathbb{R}$ such that for each $i$ as $n \rightarrow \infty, T_{f(n)}(\Sigma(i, \pm))$ converges on compact subsets of $M \times \mathbb{R}$ to a finite collection of vertical totally geodesic annuli or to a finite number of translates of $\widetilde{M}(\alpha, r)$, where $r$ and $\alpha$ are fixed. We will prove the proposition in the case where the downward translations of $\Sigma(n,+) \cup \Sigma(n,-)$ converge to vertical totally geodesic annuli. The proof of the case where the ends are asymptotic to ends of translates of $\widetilde{M}(\alpha, r)$ is quite similar, and, at the end of this section, we will indicate how to modify the arguments in this second case.

Assume now that the downward translations $T_{f(n)}$ of $\Sigma(n,+) \cup \Sigma(n,-)$ converge to a collection of vertical flat annuli.

Assertion 5.2. Let $\widetilde{\Sigma}(n)=\Sigma(1, \pm) \cup \cdots \cup \Sigma(n, \pm)$. Then, one can choose an increasing function $f: \mathbb{N} \rightarrow \mathbb{R}$ so that the sequence $T_{f(n)}(\widetilde{\Sigma}(n))$ converges smoothly on subsets of the form $M \times[-n, n]$ to a minimal lamination $\mathcal{L}$ of $M \times \mathbb{R}$ which is a product $\widehat{\mathscr{L}} \times \mathbb{R}$, where $\widehat{\mathscr{L}}$ is a geodesic lamination of $M$ by closed geodesics of uniformly bounded length. Furthermore, $f$ can be chosen so that each component of $T_{f(n)}(\widetilde{\Sigma}(n)) \cap(M \times[-3,3])$ is a normal graph over one of the annuli in $\Gamma(n) \times[-3,3]$ with sup norm less than $\frac{1}{n}$, where $\Gamma(n)$ is the collection of pairwise disjoint geodesics on $M$ associated to the ends of $\widetilde{\Sigma}(n)$ and function $f(n)$.

Proof. From our previous discussion, a diagonal argument makes it clear that a function $f(n)$ exists so that $T_{f(n)}(\widetilde{\Sigma}(n))$ converges to a vertical minimal lamination $\mathcal{L}$. By construction, $\Gamma(n)$ consists of a finite number of geodesics, since $\widetilde{\Sigma}(n)$ has only a finite number of ends. Since $\Gamma(n) \subset \Gamma(n+1)$, the closure $\widehat{\Gamma}$ of $\Gamma=\bigcup_{n=1}^{\infty} \Gamma(n)$ is a geodesic lamination of $M$ by Proposition 5.1. The assertion follows easily from these observations.

As in many of our proofs, we again would like to use the invariance of the flux of $\Sigma$ to prove that $\Sigma$ cannot have an infinite number of ends. Let $[t]$ denote the greatest 
integer less than or equal to $t$. Note that $\Sigma$ can have at most $[\operatorname{Flux}(\Sigma) / \varepsilon]$ ends where the flux is greater than $\varepsilon$. Thus, the theorem is a consequence of the next assertion.

Assertion 5.3. There exists an $\varepsilon>0$ such that for $k \in \mathbb{N}$, there exists a $K(k) \in \mathbb{N}$, such that $\Sigma(K(k))$ has at least $k$ components with flux at least $\varepsilon$.

Proof. Recall that the $\widetilde{\Sigma}(n)$ separate more and more of the ends of $\Sigma$ as $n \rightarrow \infty$. By Assertion 5.2, $T_{f(n)}(\widetilde{\Sigma}(n)) \cap(M \times[-3,3])$ consists of more and more annuli as $n \rightarrow \infty$. These annuli are converging smoothly to the annuli in $\widehat{L} \times[-3,3]$. By Assertion 5.2, there exists a leaf $\gamma \subset \widehat{L}$ such that for any fixed sufficiently small $\delta>0$, and for $W$ the $\delta$-regular neighborhood of $\gamma$ in $M$ and for $X=W \times[-3,3]$, it follows that

(1) the number of components of $T_{f(n)}(\widetilde{\Sigma}(n)) \cap(M \times[-3,3])$ contained in $X$ becomes unbounded as $n \rightarrow \infty$;

(2) the number of components of $T_{f(n)}(\Sigma(n)) \cap(M \times[-3,3])$ contained in $X$ becomes unbounded as $n \rightarrow \infty$.

Claim: There exists a small positive $\delta$ such that every compact connected minimal surface $E$ in $X$ with $\partial E$ contained in both the components of $W \times\{-3,3\}$ has flux greater than $\delta$. We prove this claim by contradiction; let $E(n)$ be such surfaces whose flux is less than $\frac{1}{n}$.

Let $E(n, *)=E(n) \cap\left(M \times\left[\frac{1}{3}, \frac{2}{3}\right]\right)$ and let $\gamma(n)$ be a least-length level set of $E(n, *)$. Without loss of generality, we will assume that

$$
\operatorname{Area}\left(E(n) \cap\left(M \times\left[h(\gamma(n)), \frac{2}{3}\right]\right)\right) \geq \operatorname{Area}\left(E(n) \cap\left(M \times\left[\frac{1}{3}, h(\gamma(n))\right]\right)\right) .
$$

With this assumption, we will make an analysis with the function $\phi=h-\frac{1}{2} d^{2}: X \rightarrow$ $[-3,3]$; in the case the opposite inequality holds, a similar analysis with the function $-h-\frac{1}{2} d^{2}: X \rightarrow[-3,3]$ will yield the desired contradiction.

Let $F(n) \subset E(n)$ be the part of $E(n)$ with boundary $\gamma(n)$ and $\phi^{-1}(2)$ and when $n$ is large and fixed, let $F=F(n)$. For $n$ large, note that $\phi^{-1}(2)=\partial F(n)-\gamma(n)$ has height approximately 2 .

A straightforward calculation shows

$$
\frac{1}{2} \Delta_{F} d^{2}=d \Delta_{F} d+\left|\nabla_{F} d\right|^{2} .
$$

Also, it is not hard to see that $\Delta_{F} d$ is uniformly bounded in $W$ for $\delta$ less than some fixed small $\delta_{0}$. Note that $\Delta_{F} \phi=-d \Delta_{F} d-\left|\nabla_{F} d\right|^{2}$. By the divergence theorem,

$$
\int_{F}-d \Delta_{F} d-\left|\nabla_{F} d\right|^{2}=\int_{F} \Delta_{F} \phi=C_{F}+\int_{\phi^{-1}(2) \cap F}\left|\nabla_{F} h-d \nabla_{F} d\right|,
$$


where $C_{F}$ is a function of $n$ that can be estimated from below by $-\operatorname{Flux}(E(n))-$ $f(n) L(\gamma(n))$; here $L(\gamma(n))$ is the length of $\gamma(n)$ and $f(n)$ is positive and tends to zero as $n \rightarrow \infty$. $C_{F}$ can be estimated this way because $C_{F}=\int_{\gamma(n)} \nabla \phi \cdot \eta=$ $-\operatorname{Flux}(E(n))-\int_{\gamma(n)} d \nabla_{E(n)} d \cdot \eta$, where $\eta$ is the outward pointing conormal to $F$, and $\left|d \nabla_{E(n)} d\right| \rightarrow 0$ as $n \rightarrow \infty$.

Next, note that for $n$ large, the following holds for some positive $K$ :

$$
\int_{F} \Delta_{F} \phi<-\frac{1}{2} \operatorname{Area}(F) \leq-\frac{1}{2} K .
$$

To see this, first note that the area $F(n)$ is greater than some constant $K$ independent of $n$, by the montonicity formula for area. Also, Flux $(E(n))<\frac{1}{n}$, so the part of $E(n)$ that makes at least any fixed positive angle with the horizontal has area going to zero as $n \rightarrow \infty$. Since $\Delta_{F} \phi$ is approximately -1 when $F$ is almost horizontal, then, for $n$ large, $\int_{F} \Delta_{F} \phi$ is approximately $-\operatorname{Area}(F)$, and so, formula (3) holds for $n$ large.

Since Flux $(E(n)) \leq \frac{1}{n}$, then, for $n$ large, the coarea formula and (1) imply,

$$
C_{F}>-\frac{1}{n}-f(n) L(\gamma(n))>-\frac{1}{n}-6 \cdot f(n) \operatorname{Area}(F) .
$$

Since $f(n) \rightarrow 0$, as $n \rightarrow \infty$, this inequality clearly contradicts equations (2) and (3).

We now briefly indicate how to modify the proof of Assertion 5.3 in the case that $T_{f(n)}(\widetilde{\Sigma}(n))$ converges to a lamination $\mathcal{L}$ of $M \times \mathbb{R}$ by translates of $\widetilde{M}(\alpha, r)$ for some fixed $\alpha$ and $r$. In this case, there exists an accumulation leaf $L$ in $\mathcal{L}$, similar to the choice of $\gamma \subset \widehat{L}$ in the previous case, such that, after a possible small translation,

(1) there is an $\varepsilon>0$ such that $\Delta=L \cap(M \times[-\varepsilon, \varepsilon])$ consists of a finite number of annuli whose tangent planes make an angle of at least $\varepsilon$ with the horizontal;

(2) if $X$ is a small regular neighborhood of $\Delta$ in $M \times[-\varepsilon, \varepsilon]$, then the number of components of $T_{f(n)}(\widetilde{\Sigma}(n)) \cap(M \times[-\varepsilon, \varepsilon])$ contained in $X$ becomes unbounded as $n \rightarrow \infty$;

(3) the number of components of $T_{f(n)}(\Sigma(n)) \cap(M \times[-\varepsilon, \varepsilon])$ contained in $X$ becomes unbounded as $n \rightarrow \infty$.

With a very small initial choice of $\varepsilon$, multiply the metric on $M \times \mathbb{R}$ by $\frac{3}{\varepsilon}$ to obtain new surfaces in the almost flat three-manifold $\widetilde{M} \times[-3,3]$, where $\tilde{M}$ is $M$ expanded by $\frac{3}{\varepsilon}$ and where the expanded set $\widetilde{\Delta} \subset \widetilde{M} \times[-3,3]$ is almost totally geodesic. Again, we define $\widetilde{X}, \phi=-h-\frac{1}{2} d^{2}$ and carry out a similar analysis as before. This discussion completes the proof of Theorem 5.1.

We now give the proof of Proposition 5.1.

Proof. Arguing by contradiction, suppose that the proposition fails. In this case, one can find an infinite collection $\Gamma=\left\{\gamma_{1}, \gamma_{2}, \ldots, \gamma_{n}, \ldots\right\}$ of distinct pairwise disjoint 
simple closed geodesics on $M$, where the length of $\gamma_{n}$ is at least 1 more that the length of $\gamma_{n+1}$.

If some $\gamma_{n}$ is homotopically non-trivial, then the geodesic or metric completion of $M-\gamma_{n}$ is a compact surface of genus $g-1$ with two closed geodesics in its boundary (in the case $\gamma_{n}$ does not separate) or consists of two compact surfaces of positive genus less than $g$, each with a single closed geodesic in its boundary. After attaching two Riemannian disks to the resulting surface, we obtain a new smooth compact Riemannian surface (possibly disconnected) with one component containing an infinite number of components of $\Gamma$ and this surface has less genus than the genus of $M$. Hence, by induction on the genus of $M$, we may assume that every geodesic $\gamma_{n}$ in $\Gamma$ bounds a compact disk $D_{n}$ in $M$.

The Gauss-Bonnet formula implies that each of the disks $D_{n}$ has total curvature $2 \pi$. Since the integral of the Gaussian curvature function on the region of $M$ where it is positive is a finite number, there is a bound on the number of such disks which are pairwise disjoint. In particular, we see that, after replacing $\Gamma$ by a subsequence, either we have an infinite chain of the form $D_{1} \subset D_{2} \subset \cdots \subset D_{n} \subset \cdots$ or the form $D_{1} \supset D_{2} \supset \cdots \supset D_{n} \supset \cdots$. If we have a chain of the second type, then replace $M$ by a smooth Riemannian surface of genus zero formed by attaching a Riemannian disk $D$ to $D_{1}$. By letting $D_{1}^{\prime}=D, D_{2}^{\prime}=D \cup\left(D_{1}-\operatorname{Int}\left(D_{2}\right)\right), \ldots, D_{n}^{\prime}=$ $D \cup\left(D_{1}-\operatorname{Int}\left(D_{n}\right)\right), \ldots$, we see that $D_{1}^{\prime} \subset \ldots \subset D_{n}^{\prime} \subset \ldots$, and so, we can always assume that we have a chain of the form $D_{1} \subset \cdots \subset D_{n} \subset \cdots$.

The closure $\bar{\Gamma}$ of $\Gamma$ is a geodesic lamination in $M$. Since the lengths of the $\gamma_{n}$ diverge, there is a limit geodesic $\alpha$ in $\bar{\Gamma}$ of a subsequence of the $\gamma_{n}$. By our chain condition on the disks $D_{n}$, the limit set $L(\Gamma)$ is disjoint from $\Gamma$, and so, $\bar{\Gamma}$ is the union of $\Gamma$ with $L(\Gamma)$. In particular, $\alpha$ is disjoint from $\Gamma$.

Let $p \in \alpha$ and identify a neighborhood of $p$ with $J \times J, J=[-1,1]$, so that $p=(0,0), J \times\{0\} \subset \alpha$, and for some decreasing sequence $y_{n} \in(0,1], y_{n} \rightarrow 0$, the segments $J \times\left\{y_{n}\right\}$ are contained in $\Gamma$. Also, choose the parametrization so that $\{0\} \times J$ is a geodesic of $M$, approximately orthogonal to the geodesic lamination.

Each disk $D_{k}$ intersects $J \times[0,1]$ in a finite number of bands, each being of the form $J \times[a, b] \subset J \times[0,1]$. Since the geodesics $J \times\left\{y_{n}\right\}$ in $\Gamma$ converge to $J \times\{0\}$, there exists a disk $D_{k_{1}}$ such that $D_{k_{1}} \cap(J \times[0,1])$ contains at least one such band and let $B_{1}$ be the band in $D_{k_{1}}$ which is lowest in $J \times[0,1]$. Again, since the geodesics $J \times\left\{y_{n}\right\}$ in $\Gamma$ converge to $J \times\{0\}$, there exists another disk $D_{k_{2}}$ such that $D_{k_{2}} \cap(J \times[0,1])$ contains a band $B_{2}$ below $B_{1}$ and such that $B_{2}$ is the lowest such band in $D_{k_{2}}$. Since the disks $\left\{D_{n}\right\}$ form a chain, the disk $D_{k_{1}} \subset D_{k_{2}}$. Continuing in this manner, we obtain an infinite ordered sequence of bands $B_{1}, B_{2}, \ldots, B_{n}, \ldots$ converging to $J \times\{0\}$.

The geodesic $\{0\} \times J$ meets each band $B_{i} \subset D_{k_{i}}$ in a geodesic $\operatorname{arc} \delta_{i}$ that separates $D_{k_{i}}$ into two closed subdisks, where we denote by $D_{k_{i}}^{\prime}$ the subdisk which is disjoint from $D_{k_{i-1}}$ (for $i=1$, one can choose either subdisks). By construction, the disks 
$\left\{D_{k_{1}}^{\prime}, D_{k_{2}}^{\prime}, \ldots, D_{k_{j}}^{\prime}, \ldots\right\}$ are pairwise disjoint in $M$. Since the boundary of each of these disks consists of two geodesic arcs with the sum of the exterior angles close to $\pi$, the Gauss-Bonnet formula implies that the total curvature of each of these disks is approximately $\pi$. Hence, in each of these disks the part of the disk with positive curvature has total curvature at least $\frac{\pi}{2}$. But then the total curvature of the part of $M$ of positive curvature is infinite. This contradiction proves that there is a bound on the lengths of the geodesics in the statement of the proposition and that the closure $\bar{\Gamma}$ of $\Gamma$ is a geodesic lamination of $M$ with the same bound on the lengths of its geodesic leaves.

\section{The unknotted theorem}

We now prove Theorem 1.4 in the Introduction. Suppose $S$ is a sphere endowed with a Riemannian metric with no stable embedded closed geodesics. We first show that $\Sigma$ is connected and has exactly two ends, one top end and one bottom end. Suppose this were not the case and $\Sigma$ had at least two top ends. By the arguments in Theorem 5.1, there would exist a stable properly embedded minimal surface $\Delta \subset S \times[0, \infty)$ with compact boundary separating the two top ends of $\Sigma$. By Theorem 4.2, either an end of $\Delta$ is asymptotic to the end of some $\widetilde{M}(\alpha, r)$ where $\alpha$ is a primitive homology class in $H_{1}(S)$ or $S$ has a stable embedded closed geodesic. Since both of these cases are excluded, $\Sigma$ must have one top end and one bottom end.

We now prove that if $\Sigma$ has finite genus, then $\Sigma$ is a Heegaard surface and unknotted. In this case, $\Sigma$ has two annular ends which we can represent by embeddings of the form $E_{+}, E_{-}: S^{1} \times[0, \infty) \rightarrow S \times \mathbb{R}$, where $\partial E_{+}$is a simple closed curve in $S \times\left\{t_{+}\right\}$ for some large positive $t_{+}$, and $E_{+}\left(S^{1} \times\{t\}\right) \subset S \times\left\{t_{+}+t\right\}$ is a simple closed curve for each $t \geq 0$; a similar statement holds for $E_{-}$, where $E_{-}\left(S^{1} \times[0, \infty)\right) \subset S \times\left(-\infty, t_{-}\right]$ and $t_{-}<t_{+}$. By elementary three-manifold theory, the ends $E_{+}, E_{-}$are standardly embedded in $S \times \mathbb{R}$. It remains to show that $\bar{\Sigma}=\Sigma \cap\left(S \times\left[t_{-}, t_{+}\right]\right)$is a standardly embedded cylinder connected sum with a standardly embedded surface of genus $g$, where $g$ is the genus of $\Sigma$. Note that the height function $h: \bar{\Sigma} \rightarrow\left[t_{-}, t_{+}\right]$has only critical points of negative index, since it is a harmonic function on $\bar{\Sigma}$. By the isotopy algorithm given in [31], any two smooth embeddings of $\bar{\Sigma}$ into $S \times\left[t_{-}, t_{+}\right]$with the same boundary values are ambiently isotopic relative to their boundary, if $h$ has no index two critical points. Since one can attach trivial handles on a standard cylinder $\bar{C} \subset S \times\left[t_{-}, t_{+}\right]$with the same boundary values as $\bar{\Sigma}$ in such a way that $h$ on the new surface only has critical points of negative index, then the original $\bar{\Sigma} \subset S \times\left[t_{-}, t_{+}\right]$ is unknotted in $S \times\left[t_{-}, t_{+}\right]$. It follows that $\Sigma$ is properly ambiently isotopic to the boundary of the solid infinite cylinder $D \times \mathbb{R} \subset S \times \mathbb{R}$ with $g$ one-handles attached in a standard way. This shows that $\Sigma$ is not only a Heegaard surface but that it is also unknotted. 
We now consider the case where $\Sigma$ has infinite genus. There are two topological possibilities for $\Sigma$, since there are exactly two different examples of orientable surfaces ( $\Sigma$ separates $S \times \mathbb{R}$ and so is orientable) with infinite genus and two ends. We now show how to embedd each of these surfaces in $S \times \mathbb{R}$ so that it is a Heegaard surface. First consider the cylinder $C=S^{1} \times \mathbb{R} \subset S \times \mathbb{R}$. Let $\Sigma_{\mathbb{N}}$ denote the surface in $S \times \mathbb{R}$ obtained by adding handles to $C$ at the points $S^{1} \times\{n\}, n \in \mathbb{N}$, and similarly define $\Sigma_{\mathbb{Z}} \subset S \times \mathbb{R}$. The surface $\Sigma_{\mathbb{N}}$ has one annular end and one end of infinite genus, and $\Sigma_{\mathbb{Z}}$ has two ends of infinite genus. Since a Heegaard surface of a non-compact three-manifold has the same end structure as the three-manifold, every Heegaard surface of $S \times \mathbb{R}$ has two ends, which proves statement (2) in Theorem 1.4. It remains to prove statements (1) and (3) when $\Sigma$ has infinite genus.

It is a classical result of Haken [12] and Waldhausen [45] that Heegaard surfaces in $S \times \mathbb{R}$ of finite genus are standard; in other words, such a Heegaard surface $H$ of genus $g$ is obtained as the boundary of $D \times \mathbb{R} \subset S \times \mathbb{R}$ with a finite number of handles attached in a standard way. Recently, Frohman and Meeks [10] were able to generalize the classical techniques of Haken and Waldhausen to prove that Heegaard surfaces in $\mathbb{R}^{3}$ are determined by their genus even when the genus is infinite. Recall that a Heegaard surface is infinitely reducible, if in the complement of any compact subdomain, there exists a proper infinite family of pairwise disjoint trivial handles on the surface. The crucial step in the proof of Frohman and Meeks is to show that a Heegaard surface of infinite genus in $\mathbb{R}^{3}$ is infinitely reducible, and this same argument can be applied in $S \times \mathbb{R}$, since each end of $S \times \mathbb{R}$ is homeomorphic to the end of $\mathbb{R}^{3}$. Thus, by the proof of uniqueness of Heegaard surfaces in $\mathbb{R}^{3}$, we obtain the similar theorem in $S \times \mathbb{R}$. This proves statement (3) of Theorem 1.4 and it remains to prove statement (1) when the minimal surface $\Sigma$ has infinite genus.

Again, in the case $\Sigma \subset S \times \mathbb{R}$ has infinite genus, we apply the techniques in [10] used to prove that a properly embedded minimal surface $M$ in $\mathbb{R}^{3}$ with one end is a Heegaard surface of $\mathbb{R}^{3}$. We now show how to adapt the arguments in [11] and [10] to our situation which is somewhat easier to control.

Let $W$ be one of the closed complements of $\Sigma$ in $S \times \mathbb{R}$. We will show that $W$ is a handlebody. Assume that $S \times\{0\}$ intersects $\Sigma$ transversely and let $W(+)=$ $W \cap(S \times[0, \infty))$ and $W(-)=W \cap(S \times(-\infty, 0])$. We will shortly show that $W(+)$, and similarly, $W(-)$ is a handlebody. Assume that this result holds. We now prove that $W$ is a handlebody. Since $W(+)$ is a handlebody with one end ( $\Sigma$ has one top end), there exists a disk $D_{+} \subset W(+), \partial D_{+} \subset \Sigma$, which separates $W(+) \cap(S \times\{0\})$ from the end of $W(+)$. Let $\widetilde{D}_{+} \subset W(+)$ be the Meeks-Yau [30] solution to the classical Plateau problem for $\partial D_{+}$in $W(+)$, i.e., $\widetilde{D}_{+}$is a least-area embedded disk in $W(+)$ with boundary $\partial D_{+}$. By the maximum principle, $\widetilde{D}_{+} \cap(S \times\{0\})=\varnothing$. Similarly, we obtain $\widetilde{D}_{-} \subset W(-)$ and let $\bar{W}$ be the closure of the bounded component of $W-\left(\widetilde{D}_{+} \cup \widetilde{D}_{-}\right)$. If $\bar{W}$ is not a handlebody, then, by the main theorem in [28], 
$\bar{W}$ contains a compact stable embedded minimal surface. This is impossible, since $\bar{W}$ is contained isometrically in $S \times \mathbb{R}$ and clearly $\bar{W}$ does not contain a minimal sphere of the form $S \times\{t\}$. It follows that $\bar{W}$ is a handlebody, and so, $W$ is a handlebody, since it is the boundary connected sum of three handlebodies. It remains to prove that $W(+)$ is a handlebody.

In order to prove $W(+)$ is a handlebody, it suffices to show that any smooth compact subdomain $\Delta \subset W(+)$ can be separated from the end of $W(+)$ by a finite collection $\mathscr{D}(\Delta)$ of pairwise disjoint disks. The proof of this reduction is a variant of the argument given in the previous paragraph, which we now repeat. Suppose $\Delta(n)$ is a smooth compact exhaustion of $W(+)$ with $[W(+) \cap(S \times\{0\})] \subset \Delta(1)$ and let $\mathscr{D}(\Delta(n))$ be the corresponding families of separating disks. Replace $\mathscr{D}(\Delta(n))$ by a collection of minimal disks of least-area in $W(+)$ with the same boundaries as in $\mathscr{D}(\Delta(n))$. By Meeks-Yau [29], this collection $\widetilde{D}(\Delta(n))$ consists of pairwise disjoint minimal disks. Note that since $h \mid(\partial \widetilde{D}(\Delta(n))) \rightarrow \infty$ as $n \rightarrow \infty$, the maximum principle implies that $h \mid(\widetilde{D}(\Delta(n))$ tends to infinity as well. Hence, we obtain a new compact exhaustion of $W(+)$ by domains $\widetilde{\Delta}(n)$ which have mean convex boundary. By the argument in the previous paragraph, $\widetilde{\Delta}(n)$ is a handlebody, but the same argument shows that the closures of the domains $\widetilde{\Delta}(n+1)-\widetilde{\Delta}(n)$ are also handlebodies. Hence, $W(+)$ is a handlebody.

Suppose there were to exist a compact $\Delta \subset W(+)$ which could not be separated from the end of $W(+)$ by a finite collection $\mathscr{D}(\Delta)$ of pairwise disjoint disks. Then, for some $n>\max (h \mid \Delta)$, for $W(n)=\{x \in W(+) \mid h(x) \geq n\}$, the compact set $W(n) \cap h^{-1}(n)$ cannot be separated from the end of $W(n)$ by a finite collection $\mathcal{D}$ of pairwise disjoint disks (since we could take $\mathscr{D}(\Delta)=\mathscr{D}$ ). Hence, it suffices to prove that $W(+) \cap(S \times\{0\})$ cannot be separated from the end of $W(+)$ by a collection $\mathscr{D}$; this is because $W(+)$ is arbitrary and so we could have replaced $\Sigma$ by a downward translation of $\Sigma$ by $n$. For $n \in \mathbb{N}$, suppose that $S \times\{n\}$ intersects $\Sigma$ transversely and consider the compact exhaustion $\Delta(n)=W \cap(S \times[0, n])$ of $W(+)$ and let $F(n)=\Delta(n) \cap \Sigma$. After a finite number of surgeries on $F(n)$ in $\Delta(n)$, we obtain a possibly disconnected incompressible surface $\widetilde{F}(n) \subset \Delta(n)$ with $\partial \widetilde{F}(n)=\partial F(n)$. From the previous paragraph, we know that for $n$ large at least one of the components of $\widetilde{F}(n)$ contains at least one boundary component in $S \times\{0\}$ and at least one boundary component in $S \times\{n\}$; otherwise, we can find the required collection of disks $\mathscr{D}$ separating $W(+) \cap(S \times\{0\})$ from the end of $W(+)$. Let $\widehat{F}(n)$ be a least-area surface in $W(+)$ in the isotopy class of $\widetilde{F}(n)$ and with $\partial \widehat{F}(n)=\partial \widetilde{F}(n)$. The third paragraph of the proof of Theorem 3.1 makes it clear that the stable surfaces $\widehat{F}(n)$ which have local curvature estimates (from stability) must have local area estimates away from their boundary. It follows that a subsequence of the $\bar{F}(n) \cap S \times[1, \infty)$ converges on compact subsets to a non-compact properly embedded stable orientable minimal surface $F$ in $S \times[1, \infty)$ with compact boundary. As observed earlier, in the first 
paragraph of this section, such an $F$ cannot exist. This contradiction proves that $W(+)$ is a handlebody, which completes the proof of Theorem 1.4.

\section{The bounded curvature theorem}

In this section, we will prove the Bounded Curvature Theorem (Theorem 1.5) stated in the Introduction. We first state a theorem that will be used to prove the Bounded Curvature Theorem.

Theorem 7.1. If $\Sigma \subset M \times \mathbb{R}$ is a properly embedded minimal surface with compact boundary and finite genus, then $\Sigma$ has bounded curvature. In particular, if $\Sigma$ is a connected properly embedded minimal surface in $M \times \mathbb{R}$ without boundary and with finite genus, then $\Sigma$ has finite topology, bounded curvature and linear area growth.

Before proving the above Theorem 7.1, we show how the first three statements in Theorem 1.5 in the Introduction follows from it. After we prove Theorem 7.1, we will finish the proof of Theorem 1.5 .

Proof of statements (1), (2) and (3) in Theorem 1.5. Statement (1) in Theorem 1.5 follows directly from the statement of Theorem 7.1. By Theorem 1.3, $\Sigma$ has finite topology, and hence, annular ends. Since $\Sigma$ is a proper minimal surface, there is a proper harmonic function (the height function) on each annular end, which implies that $\Sigma$ has finite conformal type.

Recall the hypothesis in statement (2) of Theorem 1.5 that $M$ have non-positive curvature. Since the surface $\Sigma$ in $M \times \mathbb{R}$ is minimal, it has non-positive Gaussian curvature in this case. Assume now that $M$ has non-positive curvature. Let $E \subset \Sigma$ be an annular end of $\Sigma$. Since $\Sigma$ has bounded curvature and linear area growth, there exists a sequence $b_{n} \rightarrow \infty$, so that the sequence of translated surfaces $T_{b_{n}}(E)=$ $E(n), T_{b_{n}}((p, t))=\left(p, t-b_{n}\right)$, converges to a minimal annulus $A$ properly embedded in $M \times \mathbb{R}$ with bounded curvature and linear area growth. But, since $M$ has nonpositive curvature, the minimal annulus $A$ also has non-positive curvature. Since $A$ is complete and has linear area growth, non-positive curvature, and finite topology, then it has finite total curvature $c(A)=2 \pi \chi(A)=0$. The proof that a complete Riemannian surface $\Sigma$ with non-positive curvature and linear area growth has total curvature $2 \pi \chi(\Sigma)$ is well-known (see [33] or [34] for a short proof). Thus, $A$ is a flat totally geodesic annulus in $M \times \mathbb{R}$. Hence, $A$ is either of the form $A=\gamma \times \mathbb{R}$, where $\gamma$ is an embedded geodesic in $M$, or $M$ is a flat torus and $A$ is a linear flat subannulus in $M \times \mathbb{R}$. But, if $M$ is a flat torus, it is known that the finite total curvature property of $\Sigma$ implies that the Gauss map $G: \Sigma \rightarrow S^{2}$ has finite degree and that $\Sigma$ has finite index. Thus, we may assume that $A$ is of the form $\gamma \times \mathbb{R}$ and $M$ is not a torus. 
Since $M$ has non-positive curvature, $\gamma$ minimizes length in its homotopy class. It follows that $A$ is stable. Since $M$ has non-positive curvature, it also follows that $\gamma$ is the unique geodesic in its homotopy class unless a neighborhood of $\gamma$ is flat. Let $\widetilde{M} \times \mathbb{R}$ be the covering space corresponding to $\pi_{1}(A)$ and let $\widetilde{A}$ be a lift of $A$. Since $\widetilde{A}$ is totally geodesic and the curvature of $\widetilde{M} \times \mathbb{R}$ is non-positive, the distance function to $\widetilde{A}$ is convex. An application of the maximum principle, using this convex distance function restricted to appropriate end representatives of the corresponding lift $\widetilde{E}$ of $E$ to $\widetilde{M} \times \mathbb{R}$, implies that $E$ is asymptotic to $A$. Thus, $E$ contains a subend $E^{\prime}$ which can be expressed as a small graph over the stable totally geodesic $A$. Recall that a minimal surface has finite index if and only if it is stable outside a compact set. Using the fact that the curvature of $M$ is non-positive, it is straightforward to prove that the graph $E^{\prime}$ is stable, and so, the complement of some compact subset of $\Sigma$ is stable, from which it follows that $\Sigma$ has finite index. This completes the proof of statements (1), (2), (3) of Theorem 1.5.

Proof of Theorem 7.1. Let $\Sigma$ be a properly embedded minimal surface with compact boundary and finite genus in $M \times \mathbb{R}$. By Proposition 5.1, $\Sigma$ has a finite number of ends, and so, each end of $\Sigma$ is an annulus. So, if $\Sigma$ does not have bounded curvature, then one of the annular ends of $\Sigma$ does not have bounded curvature. Thus, we may assume that $\Sigma$ is an annulus with unbounded curvature and we will derive a contradiction. Since $h: \Sigma \rightarrow \mathbb{R}$ is a proper harmonic function on $\Sigma$, after a possible reflection of $M \times \mathbb{R}$ across $M \times\{0\}$, a translation and the removal of a compact subset of $\Sigma$, we may assume that $\partial \Sigma$ is a simple closed curve in $M \times\{0\}$ and $(M \times\{t\}) \cap \Sigma$ is a simple closed curve for $t \geq 0$. The arguments which we now use to complete the proof are motivated by the proof of curvature estimates in [20] for two limit end minimal surfaces of finite genus in $\mathbb{R}^{3}$.

Arguing by contradiction, suppose that $\Sigma$ does not have bounded curvature and $p(n) \in \Sigma$ are points where the Gaussian curvature $K(p(n))$ has absolute value at least $n$. Let $T_{p(n)}: M \times \mathbb{R} \rightarrow M \times \mathbb{R}$ be the vertical downward translation by $h(p(n))$ and consider the sequence of surfaces $\Sigma(n)=T_{p(n)}(\Sigma)$ in $M \times \mathbb{R}$. After possibly choosing a subsequence, we may assume that $T_{p(n)}(p(n))$ converges to a point $p_{*} \in M \times\{0\}$.

We will first prove the theorem under the additional hypothesis that for every $p \in M \times \mathbb{R}$, there exists an $\varepsilon>0$ such that the open $\varepsilon$ ball of $M \times \mathbb{R}$ centered at $p$ intersects $\Sigma(n)$ in components that are simply connected. Then the sequence $\Sigma(n)$ is by our hypothesis uniformly locally simply connected in the sense of Colding and Minicozzi [2]. We will now discuss their theorem.

They prove a subsequence of the $\Sigma(n)$ converges to a minimal lamination $\mathcal{L}$ with singular set $S(\mathscr{L})$. The singular set $S(\mathcal{L})$ contains those points $q$ which are limit points of points of the sequence $\Sigma(n)$ where the curvature is diverging. At such a point $q$, there passes a smooth leaf $L$ of $\mathcal{L}$, and there exists a double cone $C$ with 
vertex at $q$, transverse to $L$ at $q$. Near $q$, outside of $C$, each $\Sigma(n)$ (in the convergent subsequence) contains a two component multigraph, that is converging smoothly on compact subsets of $L-\{q\}$ to $L-\{q\}$ as $n \rightarrow \infty$. Moreover the spacing between the sheets of these multigraphs is going to zero as $n \rightarrow \infty$.

Now we use this discussion at the point $q=p_{*}$, defined previously. We will prove next that the leaf $L$ through $p_{*}$ is $M \times\{0\}$. This will follow from the fact that the tangent space of $L$ is always horizontal. If not, then $T_{\widetilde{q}}(L)$ is not horizontal for some $\widetilde{q} \in L, \widetilde{q}$ near $q$. In a neighborhood of $\widetilde{q}$ there are multi-sheeted graphs of $\Sigma(n)$ converging to a fixed compact neighborhood of $\widetilde{q}$ in $L$. The number of such sheets is diverging as $n \rightarrow \infty$, and each such sheet has vertical flux uniformly bounded away from zero. This contradicts the finite flux of $\Sigma$. Thus, $L$ equals $M \times\{0\}$.

We know $S(\mathscr{L}) \cap(M \times\{0\})$ is a finite set of points containing $p_{*}$. Let $C_{i}$ be a small vertical cylinder about each singular point $p_{i}$ in $(L=M \times\{0\}) \cap S(\mathcal{L})$. Outside of the union $Q$ of the $C_{i}$, and near $L$, each $\Sigma(n)$ is a two component high sheeted, almost horizontal, multigraph we denote by $G(n)$.

Using the cyclic covering space arguments in the proof of Theorem 3.1, one can show that $G(n)-Q$ embeds in a two component infinite cyclic cover of $(M \times\{0\})-$ $Q$. Since each of the boundary curves of a component of this surface goes to a generator of $\mathbb{Z}$ under the associated represention $\sigma: \pi_{1}((M \times\{0\})-Q) \rightarrow \mathbb{Z}$ and the boundary of the component surface, which can be considered to be a commutator in the fundamental group, goes to 0 , then the number of components in $Q$ is greater than one.

Let $q$ be a point of $S(\mathcal{L}) \cap L$ that is closest to $p_{*}$ on $L$. Let $\gamma$ be a geodesic in $L$ joining $p_{*}$ to $q$. From the local picture of $\Sigma(n)$ around $p_{*}$ and $q$, for $n$ large, of a double component multigraph near $p_{*}$ and $q$, it is straightforward to construct a simple closed curve $\gamma(n)$ on $\Sigma(n)$ that consists of two almost horizontal arcs in successive sheets, which are graphs over the image of their projection to $\gamma$, together with two joining arcs near $p_{*}$ and $q$ whose lengths go to zero as $n \rightarrow \infty$ (the argument that the lengths of these arcs go to zero can be found in Assertion 1 at the end of the proof of the curvature estimates in [20] and also follows from the statement of the Lamination Metric Theorem in [36].) On the vertical cylinder $C$ near $p_{*}, \Sigma_{n}$ meets the ball bounded by $C$ in a disk $D_{n}$ and $\partial D_{n}$ consists of two double helices going from the bottom of $C$ to the top of $C$ and two arcs (on the top of $C$ and the bottom of $C$ ) joining these double helices.

The curve $\gamma(n)$ enters $C$, near height zero, at a point of one of the two helices on $C$, and then traverses $D(n)$ almost horizontally to leave $C$ at a point of the other helice on $C$. Then, $\gamma(n)$ is completed to a simple closed curve by doing the same construction in the cylinder near $q$. Let $\Gamma$ be one of the helicoidal spirals on $C \cap \Sigma(n)$ going from the bottom of $C$ to the top of $C$.

Notice that the height of $\gamma(n)$ is closer to 0 than the top and bottom of $C$. Since the height function is harmonic, any minimal disk bounded by $\gamma(n)$ has height at 
most the height of $\gamma(n)$.

Finally observe that the intersection number of $\gamma(n)$ and $\Gamma$ is one by construction. If $\gamma(n)$ were homotopic to zero on $\Sigma_{n}$, it would bound a minimal disk on $\Sigma_{n}$ which would have even intersection number with $\Gamma$, and so, $\gamma(n)$ is homotopically nontrivial.

Since $\gamma(n)$ is a simple closed curve and is homotopically non-trivial on $\Sigma(n)$ which is an annulus, the flux of $\Sigma(n)$ is equal to the flux of $\nabla h$ across $\gamma(n)$. But, by construction, the flux of $\nabla h$ across $\gamma(n)$ approaches zero as $n$ approaches infinity, which gives the desired contradiction, under our additional hypothesis that $\Sigma(n)$ is uniformly locally simply connected in the sense we described before.

The proof of the related curvature estimates in [20] is quite delicate; we will adapt the arguments given there to our situation to prove our desired curvature estimates. We now carry out the proof of the desired curvature estimates by proving the sequence $\Sigma(n)$ is uniformly locally simply connected in $M \times \mathbb{R}$.

Assertion 7.1. The sequence $\Sigma(n)$ is uniformly locally simply connected in $M \times \mathbb{R}$.

Proof. In our recent paper [24], we generalize, to the three-manifold setting, some of the key theorems of Colding and Minicozzi in [4], where they prove that a complete embedded minimal surface of finite topology in $\mathbb{R}^{3}$ is properly embedded. In fact, it follows immediately from the proof of Theorem 8.1 stated in Section 8 of this paper (the proof appears in [24]), that if the sequence $\Sigma(n)$ were not uniformly locally simply connected at $p$, then there exists a sequence of homotopically non-trivial loops $\gamma\left(n_{i}\right)$ on some subsequence $\Sigma\left(n_{i}\right)$ with lengths converging to zero. However, the fluxes of the $\Sigma\left(n_{i}\right)$ are a positive constant which is at most equal to the length of any such $\gamma\left(n_{i}\right)$, which gives a contradiction. Since this work is all very recent, we now present our original proof that the short curves $\gamma\left(n_{i}\right)$ exist.

After expansion of the metric on $M$ by a fixed constant, we may assume that the injectivity radius of $M \times \mathbb{R}$ is greater than one. For $p \in M \times \mathbb{R}$ and $r \leq 1$, let $B(p, r)$ be the open geodesic ball of radius $r$ centered at $p$. Define the function $f_{n}: M \times \mathbb{R} \rightarrow(0,1]$ to be the radius of the largest ball $B(p, r) \subset B(p, 1)$ such that $B(p, r) \cap \Sigma(n)$ consists of only simply connected components. Note the closed ball $\bar{B}\left(p, f_{n}(p)\right)$ contains a non-simply connected component when $f_{n}(p)<1$. Thus, when $f_{n}(p)<1$, there is a simple closed curve $\gamma(p, n) \subset \Sigma(n) \cap \bar{B}(p, r)$ such that $\gamma(p, n)$ does not bound a disk in $\Sigma(n) \cap \bar{B}(p, r)$.

Suppose now that the sequence $\Sigma(n)$ is not uniformly locally simply connected in $M \times \mathbb{R}$ and $p$ is a point such that $\lim _{n \rightarrow \infty} f_{n}(p)=0$. Let $p(n) \in B(p, 1)$ be points where the functions $\frac{d}{f_{n}}$ have their maximum values $\mu(n)$; here, $d: B(p, 1) \rightarrow(0,1]$ is the distance function to $\partial B(p, 1)$. Note that $B(p, 1) \subset D \times[h-1, h+1]$ where $h=h(p)$ and $D$ is the unit geodesic disk centered at $p$ in $M \times\{h\}$. After fixing the coordinates centered at $p$, we may consider the surfaces $\bar{\Sigma}(n)=\Sigma(n) \cap B(p, 1)$ to be surfaces in $D \times[-1,1] \subset \mathbb{R}^{2} \times \mathbb{R}$, where $D$ is the unit disk in $\mathbb{R}^{2}$. In 
$\mathbb{R}^{2} \times \mathbb{R}$ we consider the homothetically scaled and translated surfaces $\widetilde{\Sigma}(n)=$ $\frac{1}{f_{n}(p(n))}(\bar{\Sigma}(n)-p(n))$.

We now check that the surfaces $\widetilde{\Sigma}(n)$ form a uniformly locally simply connected sequence of planar domains in $\mathbb{R}^{3}$. After choosing a subsequence, suppose that $f_{n}(p(n)) \leq \frac{1}{2 n}$. If $q \in B\left(p(n), \frac{1}{2} d(p(n))\right)$, then $f_{n}(q) \geq \frac{1}{2} f_{n}(p(n))$. It follows from our definitions that inside the ball $B(n)$ centered at the origin of radius $n$ in $\mathbb{R}^{3}=\mathbb{R}^{2} \times \mathbb{R}, \widetilde{\Sigma}(n)$ intersects subballs of radius $\frac{1}{2}$ in simply connected components. This proves that the sequence $\widetilde{\Sigma}(n)$ forms a uniformly locally simply connected sequence of surfaces in $\mathbb{R}^{3}$. It follows from the work of Colding and Minicozzi [2], [5] that a subsequence of the $\widetilde{\Sigma}(n)$ converges to a minimal lamination $\mathcal{L}$ of $\mathbb{R}^{3}$ with singular set of convergence $S(\mathscr{L})$. We also refer the interested reader to our paper [24] for details on this argument.

Let $B(r) \subset \mathbb{R}^{3}$ denote the ball centered at the origin of radius $r$. Note that the second fundamental forms of the $\widetilde{\Sigma}(n)$ in the ball $B(2)$ can not converge to zero as $n \rightarrow \infty$, since $\widetilde{\Sigma}(n) \cap \bar{B}(1)$ is not simply connected. Thus, $\mathcal{L}$ contains a smooth leaf $L$ that is not a plane. Since $L$ is a leaf of a lamination, we know that $L \cap B(r)$ has bounded Gaussian curvature for all $r>0$.

First consider the case where $S(\mathcal{L})=\varnothing$, and let $L$ be as above. By Theorem 1.6 in [27], $L$ is properly embedded in $\mathbb{R}^{3}$, a halfspace in $\mathbb{R}^{3}$ or in a slab in $\mathbb{R}^{3}$. Since $L$ is properly embedded in a simply connected manifold, it is orientable. Since it is not a plane, we conclude by curvature estimates [43] that it is not stable. It follows that the convergence of portions of $\widetilde{\Sigma}(n)$ to $L$ is of multiplicity one, otherwise one can construct a positive Jacobi function on the limit $L$. Since the convergence of portions of the $\widetilde{\Sigma}(n)$ to $L$ is smooth with multiplicity one, a standard path lifting argument implies that $L$ has genus zero. As observed earlier, the annuli $\Sigma(n)$ are transverse to the "horizontal" foliation of $M \times \mathbb{R}$. It follows that $L$ is transverse to horizontal planes, since it has an open Gauss map and is a limit of surfaces with open Gauss map with the same property. We will show that there is a simple closed curve $\alpha$ on $L$ with non-zero vertical flux, which is a smooth limit of simple closed curves $\alpha(n) \subset \widetilde{\Sigma}(n)$. It follows, for $n$ large, that $\widetilde{\alpha}(n)$ represents a homotopically non-trivial curve on $\widetilde{\Sigma}(n)$. But then, the corresponding curves $\alpha(n)$ on $\Sigma(n)$ are homotopically non-trivial and have length converging to zero, which contradicts that $\Sigma(n)$ has fixed non-zero flux. This contradiction proves that $S(\mathscr{L})$ is non-empty. We now prove the existence of the curves $\alpha(n)$, motivated by the proof of Assertion 2 in [20].

First suppose that $L$ is properly embedded in $\mathbb{R}^{3}$. Since $\widetilde{\Sigma}(n) \cap \bar{B}(1)$ is not simply connected, $L$ is not simply connected. Since $L$ also has genus zero, $L$ has more than one end. If $L$ has finite topology, then it has finite total curvature [7]. In this case, $L$ must be a catenoid [17] which is vertical, since it is transverse to horizontal planes. But then, the waist circle of $L$ is the desired curve $\alpha$. Thus, $L$ does not have finite topology under the assumption of properness. 
So, $L$ is proper and has infinite topology. In our case of genus zero, this means that $L$ has an infinite number of ends. Since $L$ is properly embedded, the proof of the ordering theorem [9] applies to show that given any two ends of $L$, there exists an end of a plane or catenoid in $\mathbb{R}^{3}-L$ that "separates" the two ends and there is a natural linear ordering on the ends of $L$. The main theorem in [6] implies that the middle ends of $L$ in this ordering are annular ends. It follows from [7] that the middle ends of $L$ are asymptotic to ends of planes and catenoids. Since $x_{3} \mid L$ has no critical points, the ends of $L$ are asymptotic to horizontal planes. In particular, there exists a horizontal plane that intersects $L$ transversely in a finite positive number of simple closed curves (actually just one) and we let $\alpha$ be one of these simple closed curves. This completes the proof of the existence of the desired $\alpha$, which implies that $L$ is not properly embedded in $\mathbb{R}^{3}$.

Since $L$ is not properly embedded in $\mathbb{R}^{3}$, Theorem 1.6 in [27] implies that $L$ has infinite topology. In our case, this means that $L$ has an infinite number of ends. Since $L$ is properly embedded in a slab or halfspace of $\mathbb{R}^{3}$ which is a geodesically convex three-manifold, the proof of Theorem 1.6 in [27] and the proof of the ordering theorem [9] together show that given any two ends of $L$, there exists an end of a plane or catenoid in $\mathbb{R}^{3}-L$ that "separates" the two ends and there is a natural linear ordering on the ends of $L$. Now the argument procedes exactly as in the previous paragraph to obtain the desired $\alpha$. This implies that $S(\mathcal{L})$ is non-empty.

We now check that $\mathcal{L}$ is a foliation of $\mathbb{R}^{3}$ by horizontal planes with at least two vertical line components in $S(\mathcal{L})$. In fact, by Lemma 1 in [21], $\mathcal{L}$ is a foliation of $\mathbb{R}^{3}$ by horizontal planes with exactly two vertical line components. We sketch the proof for the sake of completeness.

Since the Gaussian curvature of the sequence $\widetilde{\Sigma}(n)$ is unbounded in a neighborhood of $S(\mathcal{L})$, then in some fixed ball centered at the origin, there exist points $p(n) \in \widetilde{\Sigma}(n)$ of large normalized curvature (see [27]). As shown in [27], after a translation of $\widetilde{\Sigma}(n)$ by $-p(n)$, followed by a homothety of $\widetilde{\Sigma}(n)$ by $\sqrt{K(p(n))}$, where $K(p(n))$ is the absolute Gaussian curvature at $p(n)$, we obtain a new sequence of surfaces $\widehat{\Sigma}(n)$ which converges to properly embedded simply connected surface $\Sigma$ in $\mathbb{R}^{3}$ which is transverse to horizontal planes (since the $\widehat{\Sigma}(n)$ are). By the main theorem of [27], $\Sigma$ is a vertical helicoid and it follows from [2] that $\mathscr{L}$ is a foliation by horizontal planes. By the main theorem in [35], $S(\mathscr{L})$ consists of a locally finite collection of vertical lines.

If $S(\mathcal{L})$ were to consist of a single line, then from the multisheeted graph picture of $\widetilde{\Sigma}(n)$ in any large ball for $n$ large, we would conclude that for $n$ large, $\widetilde{\Sigma}(n)$ intersects the unit ball centered at the origin in a disk, which by our normalization is not true. Thus, $S(\mathcal{L})$ consists of at least two vertical line components.

In this case, we obtain, for $n$ large, a simple closed curve $\widetilde{\alpha}(n) \subset \widetilde{\Sigma}(n)$ which consists of two almost-horizontal almost-straight-line arcs, one above the other, together with two short arcs near two singular points in $S(\mathscr{L})$ (see the discussion of 
the uniformly locally simply connected case which appears before Assertion 7.1). It follows that the corresponding curves $\alpha(n)$ in $\Sigma(n)$ are homotopically non-trivial with lengths converging to zero as $n \rightarrow \infty$. As before, this situation contradicts the fact that the flux of the $\Sigma(n)$ is non-zero. This completes the proof of the assertion, which also completes the proof of Theorem 7.1.

Proof of statements (4) and (5) in Theorem 1.5. We now complete the proof of the Bounded Curvature Theorem stated in the Introduction.

Consider a sequence $\Sigma(n)$ of minimal annuli with curvature diverging to infinity. We will prove that their fluxes converge to zero. After translation and choosing a subsequence, we may assume that the curvatures are blowing up in arbitrarily small balls in $M \times \mathbb{R}$ centered at some point $p \in M \times\{0\}$. The proof of Theorem 7.1 applies and proves that the fluxes of the $\Sigma(n)$ must be converging to zero. This proves statement (4) in Theorem 1.5.

We now prove statement (5) of the Bounded Curvature Theorem. The first part of this statement is that there exists an $\varepsilon>0$ such that when $M$ is not a sphere and $\Sigma$ is a properly embedded unstable minimal annulus in $M \times \mathbb{R}$, then the flux of $\Sigma$ is greater than $\varepsilon$. Once we prove this, curvature estimates for stable minimal surfaces and statement (4) of Theorem 1.5 imply that there is a uniform curvature estimate for all properly embedded minimal annuli in $M \times \mathbb{R}$. Although we do not use it, we remark that when $M$ has genus greater than one, then the collection of stable properly embedded minimal annuli have flux bounded from below by a positive constant.

Arguing by contradiction, suppose $\Sigma(n)$ is a sequence of properly embedded unstable minimal annuli in $M \times \mathbb{R}$ such that the flux of $\Sigma(n)$ is less than $\frac{1}{n}$.

We first consider the case where the sequence of surfaces is uniformly locally simply connected in $M \times \mathbb{R}$. From the proof of Theorem 7.1, we see that, after translating the $\Sigma(n)$ and choosing a subsequence, the $\Sigma(n)$ converge to a minimal lamination $\mathcal{L}$ of $M \times \mathbb{R}$ with $M \times\{0\}$ as a leaf of $\mathcal{L}$ and with a point $p_{*} \in S(\mathscr{L}) \cap M \times\{0\}$ in the singular set. From the proof, we know that $S(\mathcal{L}) \cap(M \times\{0\})$ consists of a finite number of points greater than 1 , and outside solid cylinders $C_{i}$ centered at the points $p_{i} \in S(\mathcal{L}) \cap(M \times\{0\})$ and near $M \times\{0\}$, the surface $\Sigma(n)$ is a highly sheeted two-component multigraph. Moreover, each component of the multigraph embeds in an infinite cyclic covering space $\pi: N \rightarrow\left((M \times\{0\})-\bigcup_{i} C_{i}\right)$.

Since the $\Sigma(n)$ are annuli, for $\varepsilon$ small the two components of $[\Sigma(n) \cap(M \times$ $[-\varepsilon, \varepsilon])]-\bigcup_{i} C_{i}$ are disks, and so, $N$ is simply connected. Since $N$ is simply connected, the fundamental group of $(M \times\{0\})-\bigcup_{i} C_{i}$ is $\mathbb{Z}$. In particular, there are two points in $S(\mathscr{L}) \cap(M \times\{0\})$ and $M$ is a sphere. Since we are assuming that $M$ is not a sphere, we obtain the desired contradiction.

We now know that the sequence $\Sigma(n)$ is not uniformly locally simply connected and, without loss of generality, we can assume that there exist points $q_{n} \in \Sigma(n)$ of diverging curvature that converge to a point $p_{*} \in M \times\{0\}$. From the proof of 
Assertion 7.1, we may assume, after small translations, that there exists a sequence $\gamma(n)=(M \times\{0\}) \cap \Sigma(n)$ of simple closed curves which have lengths going to zero as $n \rightarrow \infty$. Since the curves $\gamma(n)$ are short, we may assume also that they bound disks $D_{n} \times\{0\} \subset M \times\{0\}$. Consider the three-manifold $N(n)$, which is the closure of the component of $(M \times[0, \infty))-\Sigma(n)$ disjoint from the interior of $D_{n} \times\{0\}$. Since we are assuming that $M$ is not a sphere, $\gamma(n)$ is homotopically non-trivial in $N(n)$. Let $A(n, i)$ be a least-area annulus in $N(n)$ with boundary $\gamma(n)$ and the simple closed curve $\alpha(n, t)$ on $\Sigma(n) \cap \partial N(n)$ at height $t$. Let $A(n)$ be a limiting stable annulus for some subsequence of the $A(n, t), t \rightarrow \infty$.

Now consider $A(n)$ to lie in $M \times \mathbb{R}$. By the Stability Theorem (Theorem 1.2), either $A(n)$ is asymptotic to an end of some $M(\alpha, r)$ or under translation, the $A(n)$ produce a limit which is a vertical minimal annulus over a simple closed geodesic in $M \times\{0\}$. In the first case, we contradict that $\partial A(n)$ is homotopically trivial. In the second case, we contradict that the fluxes of the $A(n)$ are converging to zero but the flux of the limiting vertical annulus is strictly positive. These arguments complete the proof of the first part of statement (5) of Theorem 1.5 concerning curvature estimates for minimal annuli.

All of our arguments in the proof of the first part of statement (5) also apply to minimal annuli with compact boundary. Since minimal annuli with bounded curvature are quasiperiodic, it follows that the annular ends of a $\Sigma$ with finite topology have curvature bounded by any constant greater than $C_{M}$, and in particular by $2 C_{M}$, where we may assume $C_{M}$ is positive.

Remark 7.1. As noted in statement (4) of Theorem 1.5, our curvature estimate is uniform in the sense that the estimate is valid for a fixed bound of flux; the curvature estimate we obtain for $\Sigma$ only depends on a lower bound of the flux of the annulus $\Sigma$. Similarly, the curvature estimates which only depend on the vertical flux can be obtained under uniformly bounded deformations of the metric on $M$. The uniform curvature estimate in terms of flux is quite important for studying moduli space questions for minimal surfaces of genus zero in $\mathbb{R}^{3}$ and minimal annuli in $S \times \mathbb{R}$, where $S$ is the unit sphere in $\mathbb{R}^{3}$. In particular, these curvature estimates imply that minimal annuli in $S \times \mathbb{R}$ have bounded curvature and linear area growth. As explained in [22], these curvature estimates for minimal annuli can be used to prove that minimal annuli in $S \times \mathbb{R}$ with certain bounds on their horizontal (coming from the Killing fields on $S$ ) and vertical fluxes are foliated by circles; hence, these examples are in the family $\mathcal{A}$ described in Section 2.

Now we consider the case of Theorem 1.6 stated in the Introduction. The proof of this theorem is based on the same type of arguments given in the proof of Proposition 3.1; we will only briefly outline the idea of the proof. As in the proof of Theorem 7.1, the basic idea is to prove, under the hypothesis of bounded genus in 
$\varepsilon$-type horizontal "slabs" in $M \times \mathbb{R}$, that there exists a divergent sequence $\Gamma(n), \Gamma(n)$ consisting of a bounded number of simple closed curves in $\Sigma$ at approximate heights $b_{n}$ which diverge to $\infty$ or $-\infty$, such that $\Gamma(n)$ is homologous in $\Sigma$ to $\Sigma \cap(M \times\{0\})$ and such that the total length of $\Gamma(n)$ converges to zero. The existence of the $\Gamma(n)$ follows from a careful analysis of the local geometry of a properly embedded minimal surface in a Riemannian three-manifold near a point $p_{*}$ of large curvature when one has a uniform bound on the genus of the surface in a neighborhood of $p_{*}$. The local description that makes this analysis possible can be found in [5], [20] or found from a proof analysis of Theorem 7.1. In the construction of the $\Gamma(n)$, one also applies arguments similar to those used in the proof of Theorem 3.1. This completes our sketch of the proof of Theorem 1.6.

\section{Concluding remarks and conjectures}

In a recent paper, the authors have addressed the question of the role that properly embedded plays in the theory of complete embedded minimal surfaces in $M \times \mathbb{R}$. In the paper [24], we explain the structure of the closure of a complete embedded minimal surface of finite topology in $M \times \mathbb{R}$, which may or may not be properly embedded. For us, a complete embedded surface is an injective isometric immersion of a complete surface.

Theorem 8.1 (Theorem 15 in [24]). Suppose $\Sigma$ is a complete embedded minimal surface of finite topology in $M \times \mathbb{R}$. Then:

(1) The closure of $\Sigma$ is a minimal lamination of $M \times \mathbb{R}$.

(2) If $M$ has a metric of positive curvature, then $\Sigma$ is properly embedded in $M \times \mathbb{R}$.

(3) If $M$ has a metric of non-negative curvature and $\Sigma$ is not properly embedded, then $M$ is a flat torus and $\Sigma$ is a totally geodesic submanifold.

In general, there exist many complete embedded minimal surfaces of finite topology in $M \times \mathbb{R}$ which are not proper; in fact, such complete embedded minimal surfaces can even be found which are totally geodesic or which are graphs. By Theorem 8.1, a complete embedded minimal surface of finite topology in $M \times \mathbb{R}$ has locally bounded curvature and is a leaf of a minimal lamination. We believe that a better result holds for complete embedded minimal surfaces of finite genus and we make the following conjecture and question.

Conjecture 8.1. If $f: \Sigma \rightarrow M \times \mathbb{R}$ is a complete embedded minimal surface and $\Sigma$ has finite genus, then $\Sigma$ has bounded curvature. 
Question 8.1. Suppose $\Sigma$ is a complete embedded minimal surface in $M \times \mathbb{R}$. Must it be the case that $\Sigma$ has a finite number of ends? If not, will it have a finite number of ends under an additional hypothesis such as bounded curvature, finite genus, being stable or at least must $\Sigma$ have only a countable number of ends? Also, does $\Sigma$ have only a finite number of ends under some constraint on $M$ such as having positive curvature?

We mention here another important conjecture and an interesting question that arises from our work.

Conjecture 8.2. Suppose $S$ is the two-sphere endowed with a constant curvature metric. Every properly embedded minimal annulus in $S \times \mathbb{R}$ is foliated by circles of varying radii, one in each level set sphere. It then follows that a properly embedded minimal annulus in $S \times \mathbb{R}$ is either a cylinder of the form $\gamma \times \mathbb{R}$ where $\gamma$ is a great circle, a "helicoid", one of the "unduloids" defined by Pedrosa and Ritore in [37], or one of the other minimal annuli in the two-parameter family $\mathcal{A}$ defined in Section 2.2

Question 8.2. If $\Sigma$ is a properly embedded minimal surface in $M \times \mathbb{R}$, then are the annular ends of $\Sigma$ asymptotic to the ends of periodic minimal annuli in $M \times \mathbb{R}$ ?

\section{References}

[1] U. Abresch, Constant mean curvature tori in terms of elliptic functions. J. Reine Angew. Math. 374 (1987), 169-192. Zbl 0597.53003 MR 0876223

[2] T. H. Colding and W. P. Minicozzi II, The space of embedded minimal surfaces of fixed genus in a 3-manifold IV; Locally simply-connected. Ann. of Math. (2) 160 (2004), 573-615. Zbl 02157797 MR 2123933

[3] T. H. Colding and W. P. Minicozzi II, The space of embedded minimal surfaces of fixed genus in a 3-manifold III; Planar domains. Ann. of Math. (2) 160 (2004), 523-572. Zbl 02157796 MR 2123932

[4] T. H. Colding and W. P. Minicozzi II, The Calabi-Yau conjectures for embedded surfaces. Preprint.

[5] T. H. Colding and W. P. Minicozzi II, The space of embedded minimal surfaces of fixed genus in a 3-manifold V; Fixed genus. In preparation.

[6] P. Collin, R. Kusner, W. H. Meeks III, and H. Rosenberg, The geometry, conformal structure and topology of minimal surfaces withinfinite topology. J. Differential Geom. 67 (2004), 377-393.

[7] P. Collin, Topologie et courbure des surfaces minimales de $\mathbb{R}^{3}$. Ann. of Math. (2) 145 (1) (1997), 1-31. MR1432035, Zbl 886.53008. Zbl 0886.53008 MR 1432035 
[8] M. Freedman, J. Hass, and P. Scott, Least area incompressible surfaces in 3-manifolds. Invent. Math. 71 (1983), 609-642. Zbl 0482.53045 MR 0695910

[9] C. Frohman and W. H. Meeks III, The ordering theorem for the ends of properly embedded minimal surfaces. Topology 36 (1997), 605-617. Zbl 0878.53008 MR 1422427

[10] C. Frohman and W. H. Meeks III, The topological uniqueness of complete one-ended minimal surfaces and Heegaard surfaces in $\mathbb{R}^{3}$. J. Amer. Math. Soc. 10 (1997), 495-512. Zbl 0886.57015 MR 1443545

[11] C. Frohman and W. H. Meeks III, The topological classification of minimal surfaces in $\mathbb{R}^{3}$. To appear in Ann. of Math.

[12] W. Haken, Some results on surfaces in 3-manifolds. In Studies in Modern Topology 5, Englewood Cliffs, NJ, 1968, 39-98. Zbl 0194.24902 MR 0224071

[13] L. Hauswirth, Generalized Riemann examples in three-dimensional manifolds. To appear in Pacific J. Math.

[14] N. Kapouleas, Complete embedded minimal surfaces of finite total curvature. J. Differential Geom. 47 (1997), 95-169. Zbl 0936.53006 MR 1601434

[15] H. Karcher, Embedded minimal surfaces derived from Scherk's examples. Manuscripta Math. 62 (1988), 83-114. Zbl 0658.53006 MR 0958255

[16] H. Lazard-Holly and W. H. Meeks III, Classification of doubly-periodic minimal surfaces of genus zero. Invent. Math. 143 (2001), 1-27. Zbl 1031.53018 MR 1802791

[17] F. J. López and A. Ros, On embedded complete minimal surfaces of genus zero. $J$. Differential Geom. 33 (1991), 293-300. Zbl 0719.53004 MR 1085145

[18] W. H. Meeks III and J. Patrusky, Representing homology classes by embedded circles on a compact surface. Illinois J. Math. 22 (1978), 262-269. Zbl 0392.57016 MR 0474304

[19] W. H. Meeks III, J. Pérez, and A. Ros, Uniqueness of the Riemann minimal examples. Invent. Math. 133 (1998), 107-132. Zbl 0916.53004 MR 1626477

[20] W. H. Meeks III, J. Pérez, and A. Ros, The geometry of minimal surfaces of finite genus I; curvature estimates and quasiperiodicity. J. Differential Geom. 66 (2004), 1-45. Zbl 02135488 MR 2128712

[21] W. H. Meeks III, J. Pérez, and A. Ros, The geometry of minimal surfaces of finite genus III; bounds on the topology and index of classical minimal surfaces. Preprint.

[22] W. H. Meeks III, J. Pérez, and A. Ros, The geometry of minimal surfaces of finite genus $\mathrm{V}$; Jacobi fields and uniqueness for small flux. Work in progress.

[23] W. H. Meeks III and H. Rosenberg, The global theory of doubly periodic minimal surfaces. Invent. Math. 97 (1989), 351-379. Zbl 0676.53068 MR 1001845

[24] W. H. Meeks III and H. Rosenberg, The minimal lamination closure theorem. Preprint

[25] W. H. Meeks III and H. Rosenberg, Minimal surfaces of finite topology. Proceedings of the Clay Mathematics Institute Summer School on the Global Theory of Minimal Surfaces. Math. Sci. Res. Inst., to appear.

[26] W. H. Meeks III and H. Rosenberg, Stable minimal surfaces in $M \times \mathbb{R}$. J. Differential Geometry 68 (2004), 515-535. 
Vol. 80 (2005)

[27] W. H. Meeks III and H. Rosenberg, The uniqueness of the helicoid and the asymptotic geometry of properly embedded minimal surfaces with finite topology. Ann. of Math. 161 (2005), 723-754.

[28] W. H. Meeks III, L. Simon, and S. T. Yau, Embedded minimal surfaces, exotic spheres, and manifolds with positive Ricci curvature. Ann. of Math. (2) 116 (1982), 621-659. Zbl 0521.53007 MR 0678484

[29] W. H. Meeks III and S. T. Yau, The classical Plateau problem and the topology of threedimensional manifolds. Topology 21 (1982), 409-442. Zbl 0489.57002 MR 0670745

[30] W. H. Meeks III and S. T. Yau, The existence of embedded minimal surfaces and the problem of uniqueness. Math. Z. 179 (1982), 151-168. Zbl 0479.49026 MR 0645492

[31] W. H. Meeks III, The topological uniqueness of minimal surfaces in three-dimensional Euclidean space. Topology 20 (1981), 389-410. Zbl 0458.53003 MR 0617373

[32] W. H. Meeks III, The geometry, topology, and existence of periodic minimal surfaces. In Differential geometry: partial differential equations on manifolds, Proc. Sympos. Pure Math. 54, Part I, Amer. Math. Soc., Providence, RI, 1993, 333-374. Zbl 0812.49030 MR 1216594

[33] W. H. Meeks III, Geometric results in classical minimal surface theory. In Surveys in Differential Geometry 8 (S. T. Yau, ed.), International Press, Somerville, MA, 2003, 269-306. Zbl 02070186 MR 2039993

[34] W. H. Meeks III, Topological aspects of minimal surfaces in $\mathbb{R}^{3}$. In Proceedings of the 2001 Georgia Topology Conference, Proc. Sympos. Pure Math. 71, Amer. Math. Soc., Providence, RI, 2003.

[35] W. H. Meeks III, The regularity of the singular set in the Colding-Minicozzi lamination theorem. Duke Math. J. 123 (2004), 329-334. Zbl 02127998 MR 2066941

[36] W. H. Meeks III, The lamination metric for a Colding-Minicozzi minimal lamination. Illinois J. Math., to appear.

[37] R. Pedrosa and M. Ritoré, Isoperimetric domains in the Riemannian product of a circle with a simply connected space form and applications to free boundary value problems. Indiana Univ. Math. J. 48 (1999), 1357-1394. Zbl 0956.53049 MR 1757077

[38] J. Pérez and A. Ros, Properly embedded minimal surfaces with finite total curvature. In The Global Theory of Minimal Surfaces in Flat Spaces (G. P. Pirola, ed.), Lecture Notes in Math. 1775, Springer-Verlag, Berlin 2002, 15-66. Zbl 1028.53005 MR 1901613

[39] J. Pitts and H. Rubenstein, Equivariant minimax and minimal surfaces in geometric threemanifolds. Bull. Amer. Math. Soc. 19(1) (1988), 303-309. Zbl 0665.49034 MR 0940493

[40] B. Riemann, Oeuvres Mathématiques de Riemann. Gauthiers-Villars, Paris, 1898. JFM 29.0009.02

[41] H. Rosenberg, Minimal surfaces in $M^{2} \times \mathbb{R}$. Illinois J. Math. 46 (2002), 1177-1195. Zbl 1036.53008 MR 1988257

[42] H. F. Scherk, Bemerkungen über die kleinste Fläche innerhalb gegebener Grenzen. J. Reine Angew. Math. 13 (1835), 185-208.

[43] R. Schoen, Estimates for Stable Minimal Surfaces in Three Dimensional Manifolds. In Seminar on Minimal Submanifolds, Ann. of Math. Stud. 103, Princeton University Press, Princeton, NJ, 1983, 111-126. Zbl 0532.53042 MR 0795231 
[44] M. Shiffman, On surfaces of stationary area bounded by two circles, or convex curves, in parallel planes. Ann. of Math. 63 (1956), 77-90. Zbl 0070.16803 MR 0074695

[45] F. Waldhausen, Heegaard-Zerlegungen der 3-Sphäre. Topology 7 (1968), 195-203. Zbl 0157.54501 MR 0227992

Received January 28, 2003; revised September 8, 2004

William H. Meeks, III, Mathematics Department at the University of Massachusetts, Amherst, MA 01003, U.S.A.

E-mail: bill@gang.umass.edu

Harold Rosenberg, Mathematics Department at the University of Paris VII, Paris, France

E-mail: rosen@math.jussieu.fr 